\title{
Article \\ Numerical Investigation of an Optimized Rotor Head Fairing for the RACER Compound Helicopter in Cruise Flight
}

\author{
Patrick Pölzlbauer ${ }^{1, *(1)}$, Andreas Kümmel ${ }^{1}\left(\mathbb{D}\right.$, Damien Desvigne ${ }^{2}$ and Christian Breitsamter ${ }^{1}$ \\ 1 Chair of Aerodynamics and Fluid Mechanics, Technical University of Munich, 85748 Garching, Germany; \\ andreas.kuemmel@tum.de (A.K.); christian.breitsamter@aer.mw.tum.de (C.B.) \\ 2 Department of Aerodynamics, Airbus Helicopters, 13700 Marignane, France; damien.desvigne@airbus.com \\ * Correspondence: patrick.poelzlbauer@tum.de; Tel.: +49-89-289-16132
}

Citation: Pölzlbauer, P.; Kümmel, A.; Desvigne, D.; Breitsamter, C.

Numerical Investigation of an Optimized Rotor Head Fairing for the RACER Compound Helicopter in Cruise Flight. Aerospace 2021, 8, 66. https://doi.org/10.3390/

aerospace 8030066

Academic Editor: Rosario Pecora

Received: 3 February 2021

Accepted: 3 March 2021

Published: 5 March 2021

Publisher's Note: MDPI stays neutral with regard to jurisdictional claims in published maps and institutional affiliations.

\begin{abstract}
The present work is part of the Clean Sky 2 project Full-Fairing Rotor Head Aerodynamic Design Optimization (FURADO), which deals with the aerodynamic design optimization of a fullfairing rotor head for the Rapid And Cost-Effective Rotorcraft (RACER) compound helicopter. The rotor head is a major drag source and previous investigations have revealed that the application of rotor head fairings can be an effective drag reduction measure. As part of the full-fairing concept, a new blade-sleeve fairing was aerodynamically optimized for cruise flight. Within this publication, the newly developed blade-sleeve fairing is put to test on an isolated, five-bladed rotor head and compared to an already existing reference blade-sleeve fairing, which was developed at Airbus Helicopters. Numerical flow simulations are performed with ANSYS Fluent 2019 R2 considering a rotating rotor head with cyclic pitch movement. The aerodynamic forces of the isolated rotor head are analyzed to determine the performance benefit of the newly developed blade-sleeve fairing. A drag reduction of $4.7 \%$ and a lift increase of $20 \%$ are obtained in comparison to the Airbus Helicopters reference configuration. Furthermore, selected surface and flow field quantities are presented to give an overview on the occurring flow phenomena.
\end{abstract}

Keywords: RACER; compound helicopter; rotor-head aerodynamics; FURADO; Clean Sky 2; CFD; helicopter aerodynamics

\section{Introduction}

Nowadays, the aviation industry enables a seamless door-to-door mobility by multiple transportation means and the number of transported passengers has significantly increased within the last decade [1]. Due to the large traffic volume, the environmental impact of the aviation industry has become a major topic for society. This has caused the engineers to strive for environmentally friendly aircraft, which exhibit reduced greenhouse gas emissions as well as a low noise footprint. In order to push the development of innovative technologies in aviation industry, Europe has defined ambitious goals in the agenda of Flightpath 2050 [2] and set up initiatives financing common research projects. Within the Clean Sky 2 joint undertaking [3], novel aircraft configurations and capabilities are explored for different aircraft categories. Rotorcraft play an important role in terms of seamless mobility, since they do not require a large ground infrastructure and they can perform vertical takeoff and landing. This represents an important capability for several mission scenarios. Helicopters are typically used for Helicopter Emergency Medical Service (HEMS), Search And Rescue (SAR), passenger transport and parapublic missions. All of these scenarios have in common that time is a critical factor, which demands for high speed helicopters. Therefore, the development of future helicopters is subject to the reduction of the environmental impact on the one hand and the expansion of the flight envelope towards higher cruising speeds on the other hand. There are two main reasons for the speed limitation of a conventional helicopter. These are given by the maximum permissible tip speed of the advancing rotor blade, in order to avoid transonic effects, and the retreating 
blade stall, which causes a lift dissymmetry on the rotor [4]. This physical limitation can only be tackled by changing the conceptual design of the helicopter. Within Clean Sky 2, the cruise speed limitation is addressed by the Fast Rotorcraft Innovative Aircraft Demonstrator Platforms (IADP). One of the demonstrators is represented by the Rapid And Cost-Effective Rotorcraft (RACER) [5]. The RACER compound helicopter, which can be seen in Figure 1, is developed under the lead of Airbus Helicopters, together with European industrial and academic partners.



Figure 1. Rapid And Cost-Effective Rotorcraft (RACER) [5].

It combines the beneficial characteristics of fixed-wing aircraft and rotorcraft by providing vertical takeoff and landing capabilities and enabling fast and efficient forward flight. Considering an advanced helicopter design, there are two possibilities to increase the speed of the helicopter, namely to provide more power or to reduce the drag. Therefore, drag reduction has always been an important topic during the helicopter development. The relevance of drag mitigation is reflected by the large number of experimental and numerical investigations that have been performed on this topic within the last decades. Stalewski [6] and Batrakov et al. [7] performed an aerodynamic design optimization of the helicopter rear fuselage with respect to drag reduction. For this purose, they used numerical flow simulation in combination with a genetic optimization algorithm to modify the parameterized shape of the helicopter backdoor region. Furthermore, comprehensive experimental investigations were conducted by Le Pape et al. [8] and De Gregorio [9] using active flow control in the backdoor region of a helicopter model to decrease the fuselage drag by alleviating the flow separation. In the Aerodynamic Design Optimization of a Helicopter Fuselage including a Rotating Rotor Head (ADHeRo) project [10,11], experimental and numerical investigations were performed for a Twin Engine Light (TEL) class utility helicopter providing detailed flow characteristics and drag analysis. Furthermore, the impact of passive flow control devices, like strakes and vortex generators, on the fuselage drag of a helicopter was investigated by Grawunder et al. [11] and Boniface [12]. Besides the fuselage, the rotor head of a helicopter represents a major drag source, which has been indicated by several drag breakdown studies [13-15]. Depending on the weight class and the cruise speed of the helicopter, the rotor head roughly contributes between 25 and 40 percent of the total parasite drag. Khier [16] investigated interference effects between the rotor hub and the fuselage of a conventional helicopter by numerical flow simulations in order to identify drag reduction potential.

Moreover, Desvigne [17] performed numerical flow simulations accompanying windtunnel measurements in the European Contribution to Analysis of Rotor-hub Drag reduction (CARD) project, which dealt with the investigation of different combinations of pylon fairings, blade-sleeve fairings and hub-cap designs to assess drag-reduction capabilities. Graham et al. [18] and Martin et al. [19] investigated advanced hub and pylon fairing 
configurations in the wind tunnel of the Ames Research Center. Their main objectives were to determine interference effects between the rotor head fairings and to identify further drag reduction potential by the application of well designed fairing shapes. Regarding drag reduction of a heavy load class transport helicopter, Wentrup et al. [20] conducted numerical investigations aimed at optimizing the surface of the fuselage backdoor and reducing the rotor head drag by installing a hub fairing. The numerical flow simulations revealed a drag reduction potential between 13 and $23 \%$ of the total helicopter drag. The present work is part of the Full-Fairing Rotor Head Aerodynamic Design Optimization (FURADO) project, which is well aligned with the required innovation for high-speed rotorcraft. The project is aimed on the aerodynamic design optimization and flow analysis of a semi-watertight full-fairing rotor head with respect to drag reduction of the RACER compound helicopter [21-23]. Figure 2 shows the comparison between a fully articulated Airbus Helicopters H175 rotor head without fairings and the RACER full fairing. Within FURADO, a special focus was set on the aerodynamic design optimization of the bladesleeve fairing (BSF), the full-fairing beanie (FFB) and the pylon fairing for a sea-level cruise flight with 220 knots. The blade-sleeve fairing, which is shown by the orange component in Figure 2, was aerodynamically optimized within the FURADO project. For this purpose, a database of optimized airfoil shapes was developed for selected radial sections of the fairing [22].


Figure 2. Airbus Helicopters H175 rotor head without fairings [24] (left) and RACER full fairing concept (right).

Moreover, the best performing airfoils were used as the basis for the three-dimensional design optimization using a genetic optimization algorithm [23]. The objective functions were to reduce the drag on the retreating rotor blade and to increase the lift-to-drag ratio on the advancing rotor blade. For the present work, the blade-sleeve fairing design offering the best compromise between the two objective functions was chosen to be investigated on the isolated, rotating rotor head. In order to be able to determine the benefit of the newly developed fairing, it is compared to a reference geometry, which was developed by Airbus Helicopters during the preliminary design phase of the RACER demonstrator.

\section{Numerical Setup}

Within this section, the investigated geometry, the mesh generation process, the flow simulation setup and the deployed computational resources are presented. Furthermore, the methods applied to realize the rotor blade pitch movement are explained giving a comprehensive overview on the numerical approach.

\subsection{Model}

The investigated geometry represents a full-scale model of the isolated, five-bladed RACER rotor head, which is illustrated in Figure 3. The main focus of the present work lies on the evaluation of the aerodynamic forces of the rotor hub. Therefore, the model does not include the full main rotor and the rotor blades are truncated at about one third of the rotor radius. It is quite common in wind-tunnel measurements to use truncated rotor blades in fuselage-tail section configuration testing [10]. Therefore, using a numerical 
model with truncated rotor blades enables a direct comparison to experimental data. Based on the experience of previous research, the predicted hub drag of a wind-tunnel model with truncated rotor blades is still representative. The investigated model consists of the full-fairing beanie (FFB) (green), the blade-sleeve fairing (BSF) (orange), the truncated rotor blades (blue), a spherical junction between the fairings (red) and the lead-lag dampers (violet). All gaps between the fairings are closed yielding a fully watertight geometry for the mesh generation.

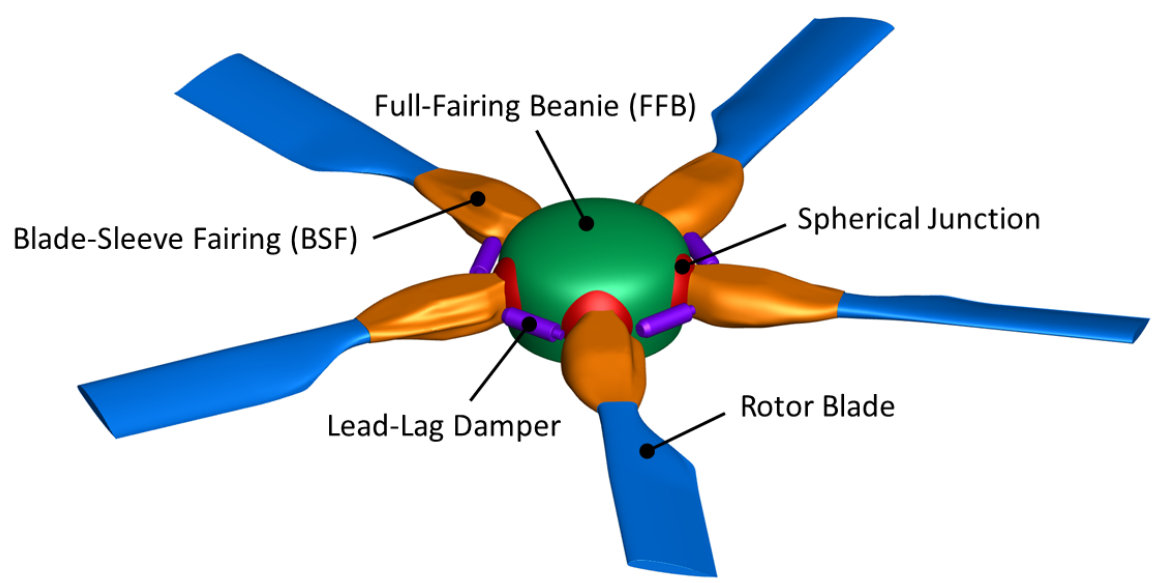

Figure 3. Components of the isolated rotor head model.

\subsection{Mesh Generation}

The present flow problem features a rotating rotor head with cyclic pitch movement, which means that the pitch angles of the rotor blades are continuously changing over one rotor revolution. In order to realize the cyclic pitch movement, an overset mesh approach is employed in combination with mesh motion. Before the applied computational grid is presented, an introduction to overset mesh interpolation is given.

\subsubsection{Overset Mesh Interpolation}

Overset mesh, also known as Chimera, allows to subdivide a computational domain into several overlapping regions, which can be independently meshed from each other. Therefore, the mesh generation effort for complex geometries can be significantly reduced. The Chimera technique was first introduced by Benek et al. [25] in 1983. The principle idea of overset mesh is to connect different overlapping cell zones by an overset interface, which allows to interpolate the cell data at the newly formed cell zone boundaries. Figure 4 shows an exemplary 2D overset mesh case including three component grids. It can be seen that all three component meshes are embedded in the background mesh overlapping each other. Moreover, the blue cell zone is protruding the boundary of the background mesh at the bottom. The wall boundaries of the mesh components and the overset boundaries are marked in Figure 4a. According to Benek et al. [26], a hierarchical embedding process is applied during the overset mesh generation to manage the data among the grids. Embedded grids are on a lower hierarchical level than the grids in which they are embedded. Therefore, the highest level would be occupied by the background mesh and the remaining component meshes are on the same hierarchical level. The application of such an approach provides advantages in the generation of the data structure and the limitation of searches necessary to find points in other grids, which may be used for the interpolation of boundary data. Regarding Figure $4 \mathrm{a}$, it can be seen that some points of the background mesh lie within the solid boundary of the component meshes. These points are located outside of the computational domain and must be excluded from the solution process. 


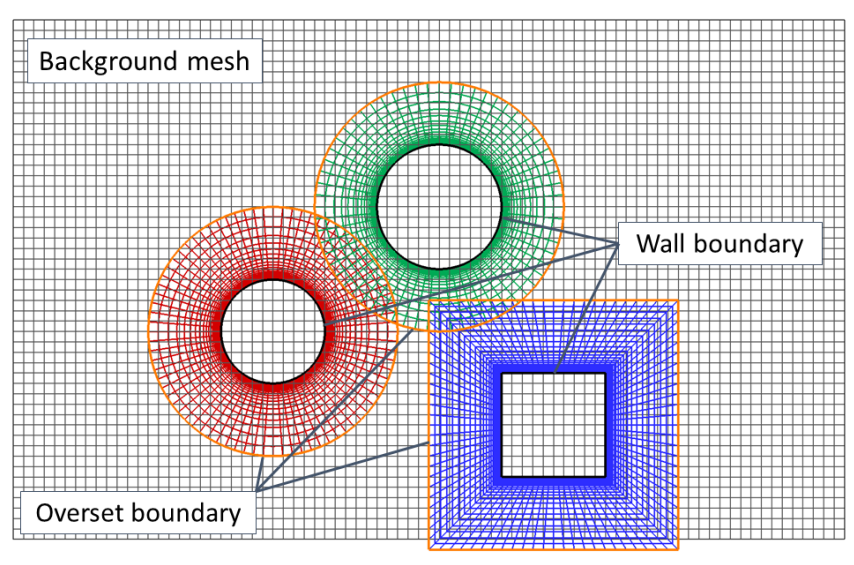

(a) Background mesh and three embedded component meshes.

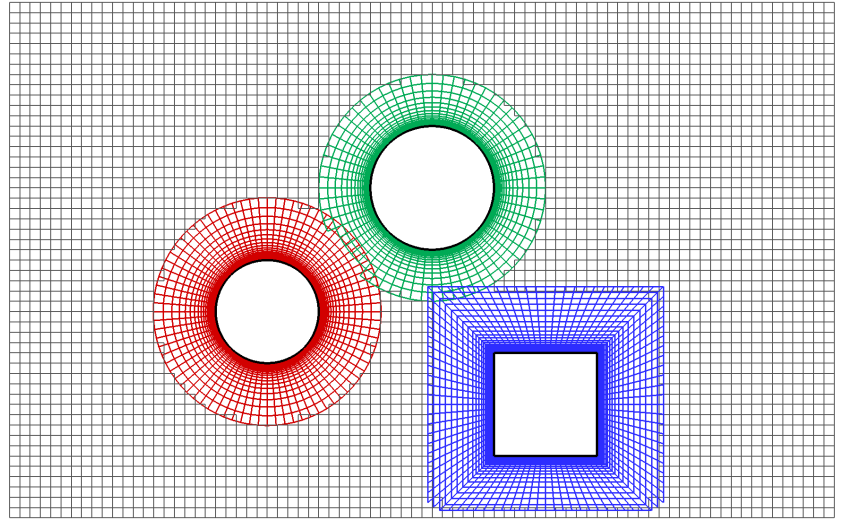

(b) Solve cells after the initialization of the overset mesh.

Figure 4. Exemplary visualization of the overset mesh generation using a background mesh with three component meshes.

In general, if no points would be discarded and the background mesh has a lot of points in commmon with the component mesh, a significant amount of grid data would have to be interpolated and updated, which is time consuming. Moreover, Lombard and Venkatapathy [27] stated that an extensive interpolation between the meshes can degrade the global accuracy, especially when there is a large discrepancy in the spatial resolution. In order to avoid such problems, only the boundary of each embedded grid is updated.

The exclusion of certain cells from the solution process is called hole cutting. For the given 2D example, the initialized overset mesh after hole cutting is depicted in Figure $4 \mathrm{~b}$. In the final mesh, regions of different cell types are present, which can be seen in Figure 5. On the left hand side of Figure 5, the background mesh is shown after hole cutting. Moreover, the corresponding component meshes are illustrated on the right hand side. The blue colored regions correspond to blanked/masked cells, which are discarded from the solution process on the respective grid level. Furthermore, there are computed cells given in green, which are applied to calculate the flow equations. Depending on the interpolation method between the different sub grids, a certain overlap must be ensured to enable an interpolation at the hole boundary. The communication between the background and the component meshes is achieved by donor (red) and receiver (cyan) cells. The donor cells constitute a subset of the computed cells and provide data to the receiver cells of the neighboring domain. In order to reduce interpolation errors at the overset boundaries, a similar mesh resolution should be provided in the transition region between the mesh components.

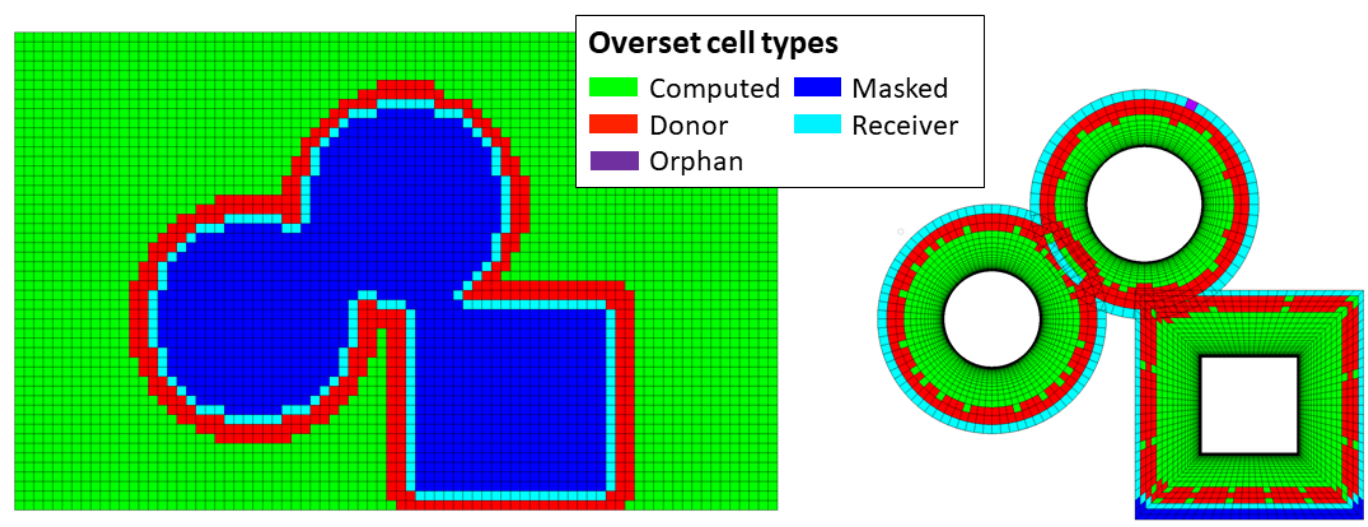

Figure 5. Overset mesh cell types and visualization of the hole cutting process forming a new boundary used for data interpolation between the mesh components.

The final step in setting up the overset mesh connectivity is the donor search. For this purpose, valid compute cells are determined, which contain the cell centroids of the 
corresponding receiver cells. At least one valid donor cell is required for each receiver. According to the ANSYS Fluent user guide [28], four or more cells are required in the overlap region of both meshes to enable a successful donor search. Finally, the receiver cells form the fringe layer of a mesh zone defining the transition region to the opposing mesh. A valid mesh overlap is depicted in Figure 6 for two component meshes. If the donor search fails and the receiver cell cannot find a valid donor, it becomes an orphan cell. The presence of orphan cells indicates an insufficient overlap between the meshes or a too large discrepancy in the mesh resolution.

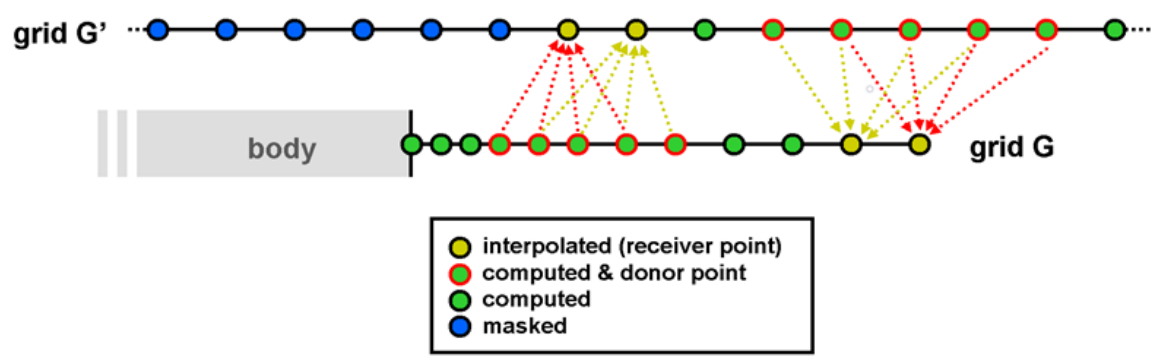

Figure 6. Sketch of a valid mesh overlap leading to a successful donor search for two mesh components [15].

\subsubsection{Computational Grid}

As mentioned, an overset mesh approach is applied to realize the numerical flow simulations. The mesh consists of several subdomains, which are intersected and combined within the flow simulation process. The rotational symmetry of the five-bladed rotor head is exploited and only one fifth of the computational grid must be generated. Block-structured hexahedral meshes are created with ANSYS ICEM CFD 2019 R2 providing high quality grids. Figure 7 shows the size and the blocking of the far-field domain. Here, the quantity $\mathrm{R}$ corresponds to the rotor radius of the investigated rotor head with truncated blades. The domain has a length of $30 \mathrm{R}$ as well as a width and a height of $15 \mathrm{R}$. The box in the middle of the far-field domain yields the background mesh of the rotor head, which is connected to the outer domain via a mesh interface. The embedded rotor head mesh consists of 16 component grids, which all feature a resolved boundary layer. The initial cell height is selected to provide a dimensionless wall distance of $y^{+} \approx 1$.

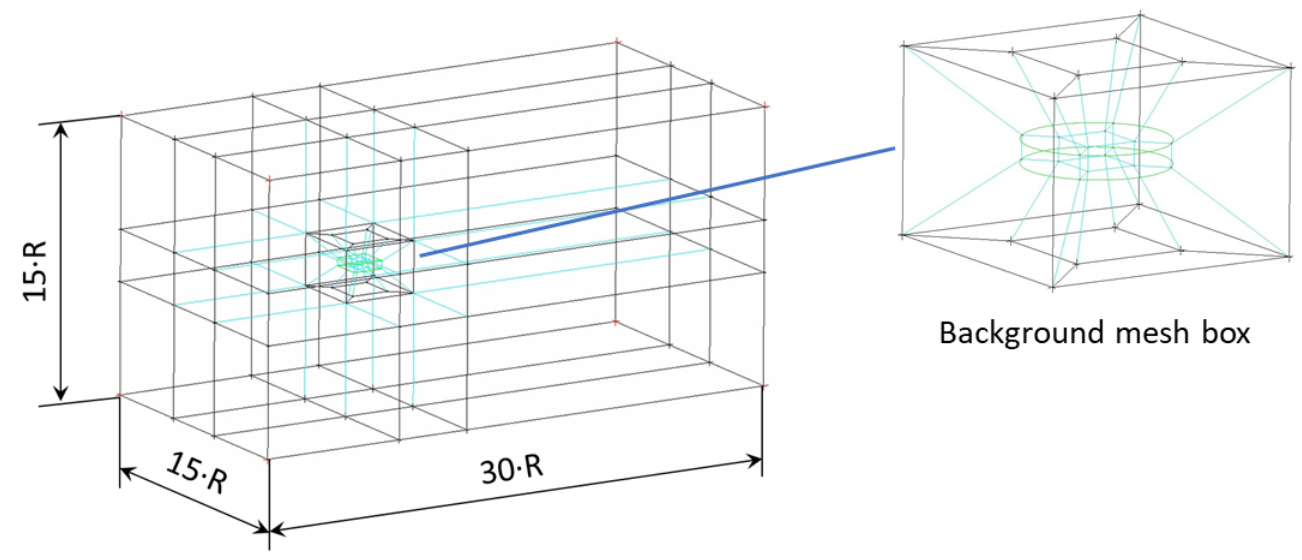

Figure 7. Size of the far-field domain with the overset background mesh in the middle of the domain.

The blocking strategies for the beanie and the rotor blade are illustrated in Figure 8 . Regarding the beanie mesh, periodicity is applied at the cell vertices of the two symmetry planes. Therefore, a proper mesh alignment can be ensured at the periodic boundaries. 


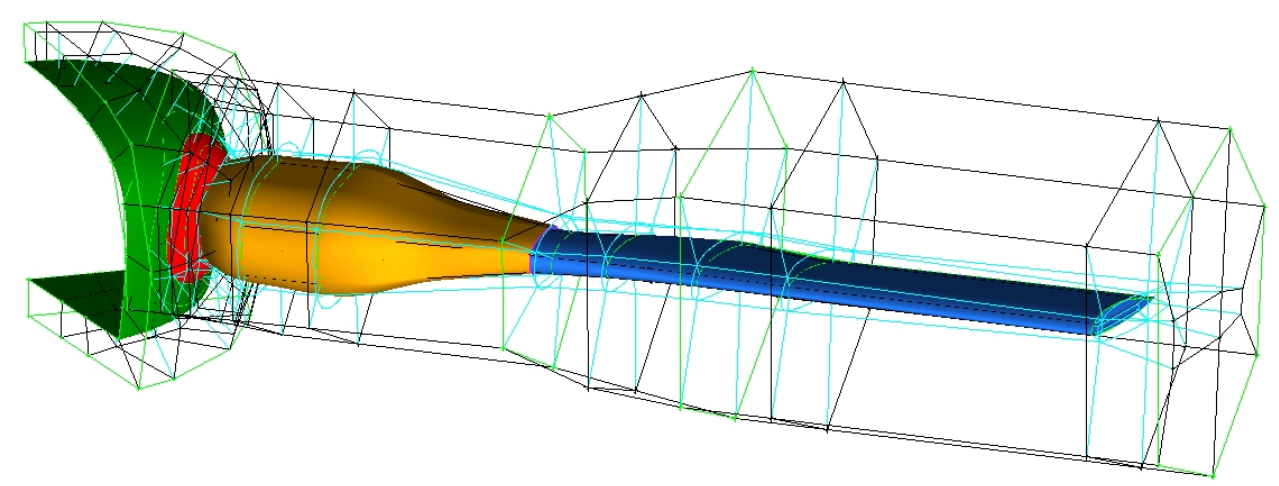

Figure 8. Blocking strategy of the rotor head components demonstrated by the beanie and the rotor blade mesh domain.

The present flow problem has intersecting wall boundaries between the spherical junction of the rotor blade and the beanie. Intersecting geometries are not allowed within the applied overset mesh approach. Therefore, a collar mesh is required to connect the intersecting components preserving the boundary layer mesh. The domain of the collar mesh is depicted in Figure 9 and a detailed view on the surface mesh is given. It is important that the same cell sizes are applied in the overlapping region and that the initial cell heights are identical to avoid orphan cells.

In order to determine the influence of the spatial discretization on the flow simulation results, a grid resolution study was conducted for the rotating rotor head with cyclic pitch movement. The investigated flight condition corresponds to the RACER cruise flight at sea-level. Three different mesh resolutions with 13, 29 and 52 million elements are considered and the mean aerodynamic forces are compared to each other. The results of the grid resolution study are summarized in Table 1. The differences of lift and drag are given relative to the finest mesh. It can be seen that the medium sized mesh is slightly overpredicting lift with $1.9 \%$ and that the obtained drag differs by $0.7 \%$. Regarding the coarse mesh, the difference in lift is $1.2 \%$ and the obtained drag is $7.8 \%$ higher than for the finest mesh. The main purpose of the grid sensitivity study was to find a reasonable mesh size offering a good compromise between numerical accuracy and computational effort.

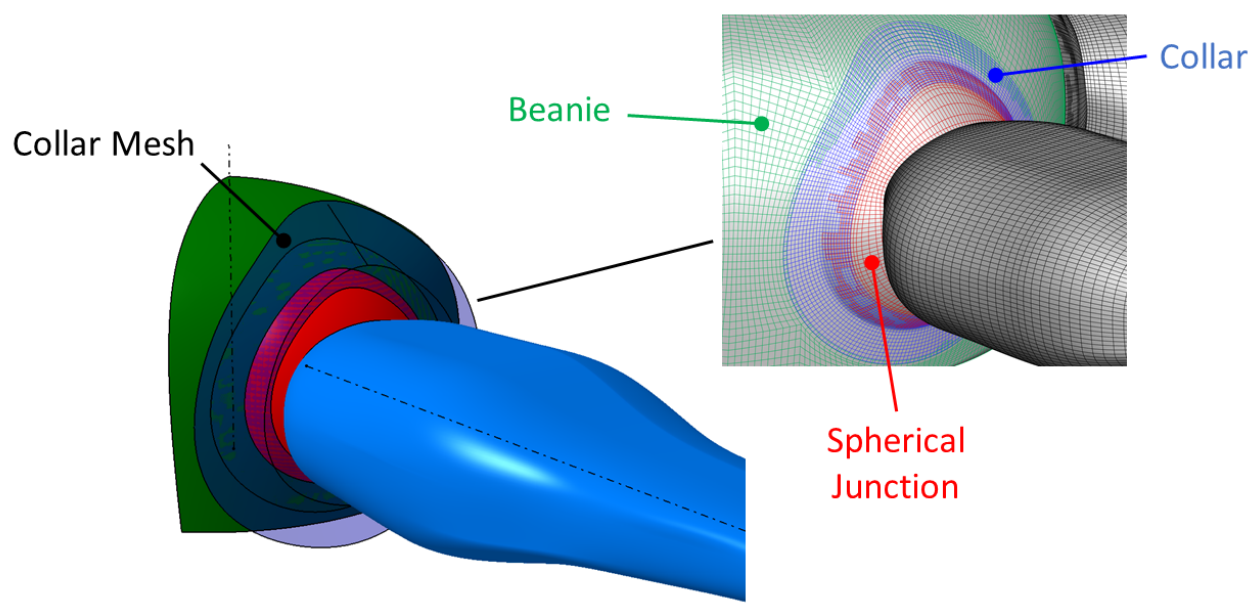

Figure 9. Definition of the collar mesh enabling a valid transition between the beanie and the rotor blade mesh.

The medium sized grid only differs by less than $1 \%$ concerning drag and less than $2 \%$ concerning lift in comparison to the finest mesh with 52 million elements. Considering the highly unsteady loads acting on the rotor head, this represents a good agreement between the two meshes and satisfies the quality requirements. The fact that lift is not perfectly 
converging to the value of the fine mesh is not decisive for the outcome of the present work. The focus of the numerical investigations is on the comparison of the aerodynamic forces of two different rotor head fairing configurations using a computational mesh with the same spatial resolution. Therefore, only relative differences between the two cases are of primary interest and the error in the absolute forces becomes less important.

Table 1. Mesh resolution study for the isolated rotor head.

\begin{tabular}{llll}
\hline Mesh Size & No. of Elements $\left(\times \mathbf{1 0}^{\mathbf{6}}\right)$ & $\mathbf{\Delta}$ ift $(\mathbf{\%})$ & $\Delta$ Drag $(\mathbf{\%})$ \\
\hline Fine mesh & 52 & - & - \\
Medium mesh & 29 & +1.9 & -0.7 \\
Coarse mesh & 13 & +1.2 & +7.8 \\
\hline
\end{tabular}

Table 2 gives an overview on the mesh sizes of the different component grids, which are all discretized by hexahedral elements. Overall, the mesh consists of 1205 blocks with 29 million elements. The background mesh comprises almost half of the mesh size, because the size of the elements in the cylindrical domain containing the rotating rotor head must be kept low to avoid the generation of orphan cells at the overset boundaries.

Table 2. Summary of the component mesh sizes.

\begin{tabular}{lll}
\hline Cell Zone (Domain) & No. of Blocks & No. of Elements $\left(\times \mathbf{1 0}^{\mathbf{6}}\right)$ \\
\hline Farfield & 26 & 1.28 \\
Background mesh & 19 & 13.05 \\
Beanie & 240 & 2.24 \\
Rotor blade 1-5 & $142 \times 5$ & $2.20 \times 5$ \\
Collar 1-5 & $16 \times 5$ & $0.15 \times 5$ \\
Damper 1-5 & $26 \times 5$ & $0.14 \times 5$ \\
\hline Total & 1205 & 29.03 \\
\hline
\end{tabular}

A detailed view on the applied computational mesh is given in Figure 10. Additionally, the overset cell types of the background mesh are visualized after hole cutting showing the boundaries of the component grids by the colored donor and receiver cells.

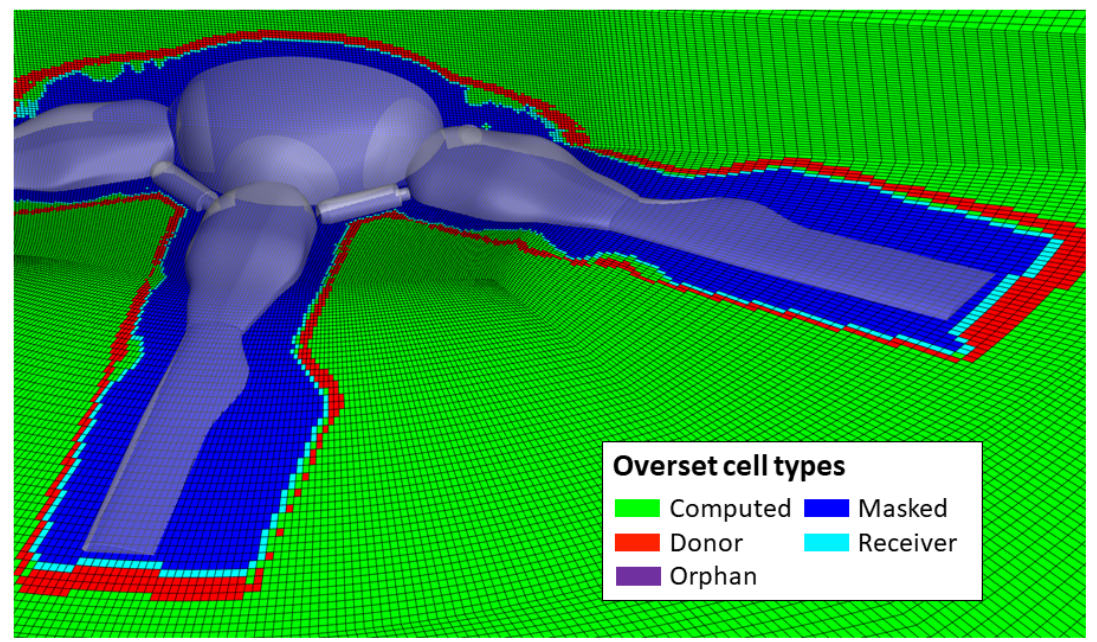

Figure 10. Slices through the applied computational mesh of the isolated rotor head showing the overset mesh cell types. 


\subsection{Blade Pitch Movement}

Within the numerical flow simulations, the actual pitch angles of the rotor blades are applied according to a trimmed sea-level cruise flight of the RACER demonstrator with 220 knots. The rotor is rotating in clockwise direction and the definition of the azimuthal rotor blade position is illustrated in Figure 11. Here, a downwind pointing rotor blade corresponds to $\Psi=0^{\circ}$. The controls of the helicopter main rotor comprise the collective and the cyclic pitch input. The definition of the actual blade pitch angle $\theta(\Psi)$ is given by Equation (1). The collective pitch $\theta_{0}$ is used to change the blade pitch angles of all rotor blades simultaneously, which defines the average lift generated by the main rotor. Furthermore, the cyclic pitch input enables the flight attitude control of the helicopter in the three-dimensional space. It consists of the longitudinal cyclic pitch $\theta_{1, s}$ and the lateral cyclic pitch $\theta_{1, c}$, which realize the pitch and roll movement, respectively. The implemented version of the general blade-pitch law, which is used within the numerical flow simulations, is shown by Equation (2). The rotational speed $\omega$ and the simulation time $t$ define the time-dependent azimuthal rotor blade position $\Psi$. Furthermore, a phase lag of $72^{\circ}$ between the rotor blades must be considered for a five-bladed rotor head. If mesh motion should be used within the flow simulations, the blade-pitch velocity is required, which is defined by Equation (3). The flapping and the lead-lag movement of the rotor blades due to the unsteady aerodynamic loads are neglected within the present investigations.



Figure 11. Definition of the azimuthal rotor blade position.

General blade pitch law:

$$
\theta(\Psi)=\theta_{0}+\theta_{1, s} \cdot \sin (\Psi)+\theta_{1, c} \cdot \cos (\Psi)
$$

Implemented blade pitch law:

$$
\begin{gathered}
\theta(\omega, t, n)=\theta_{0}+\theta_{1, s} \cdot \sin (\omega t+n \Delta \Psi)+\theta_{1, c} \cdot \cos (\omega t+n \Delta \Psi) \\
\dot{\theta}(\omega, t, n)=\frac{d \theta}{d t}=\theta_{1, s} \cdot \omega \cdot \cos (\omega t+n \Delta \Psi)-\theta_{1, c} \cdot \omega \cdot \sin (\omega t+n \Delta \Psi)
\end{gathered}
$$

Five rotor blades: $n=\{0,1,2,3,4\}$ and $\Delta \Psi=72^{\circ}$

\subsection{Flow Solver}

The numerical flow simulations of the isolated RACER rotor head are conducted with ANSYS Fluent 2019 R2. The investigated flight condition corresponds to a sea-level cruise flight of the RACER demonstrator in ICAO standard atmosphere with $V_{\text {Cruise }}=220 \mathrm{kts}$. The compressible, unsteady Reynolds-Averaged Navier-Stokes equations (URANS) are solved using the pressure-based, coupled solver of ANSYS Fluent. The pressure interpolation is achieved by the standard pressure scheme. Turbulence modeling is realized by the two-equation k- $\omega$ SST model [29]. Regarding the spatial discretization, a bounded central differencing scheme is used for momentum and second order upwind schemes are chosen for the remaining quantities. Further, a least-square cell-based formulation is 
applied for the gradient calculation and a bounded second-order implicit scheme is used for the temporal discretization. Pressure far-field boundary conditions are applied on each side of the far-field domain with a free-stream Mach number of $M_{\infty}=0.33$. According to the ICAO standard atmosphere, the ambient pressure is $p_{\infty}=1013.25 \mathrm{hPa}$ and the ambient temperature is $T_{\infty}=288.15 \mathrm{~K}$. The turbulence quantities at the far-field boundaries are given by a turbulence intensity of $T u=1 \%$ and a turbulent viscosity ratio of $\mu_{t} / \mu=10$. The overset mesh consists of 16 components and one background mesh. Furthermore, the background mesh is connected to the far-field domain via a mesh interface. At the beginning of the flow simulation, the correct pitch angles are applied to each of the five rotor blades for one blade pointing upstream. For this purpose, dynamic mesh is used in combination with a user-defined function (UDF) containing the blade pitch law according to Equation (2). The collective and cyclic pitch inputs required for a trimmed flight condition are provided by Airbus Helicopters. The flow simulation is globally initialized with constant values for pressure, velocity, turbulent kinetic energy, specific dissipation rate and temperature. Thereafter, a steady state solution is calculated for the non-rotating rotor head, which serves as the input for the transient simulation. Mesh motion is applied to realize the movement of the rotor head components. For this purpose, a UDF is defined for the beanie and the five rotor blades. The cell zones of the rotor blades, the collar grids and the lead-lag dampers move relative to the beanie domain. The selected time-step size is according to $1^{\circ}$ of azimuth angle per time step, which provides good convergence behavior and enables a successful overset mesh generation. For each time step, about 30 inner iterations are required to reach convergence. The highest remaining scaled residual is given by the continuity residual, which is reduced by three orders of magnitude.

\subsection{Computational Performance}

The numerical flow simulations of the isolated RACER rotor head have been performed on the SuperMUC-NG of the Leibniz Supercomputing Centre. The computations have been parallelized on seven Intel Xeon Platinum 8174 nodes with 336 cores resulting in an average load of 86.000 cells per processor. A full rotor revolution with 360 time steps has been accomplished within $16 \mathrm{~h}$. The applied numerical approach with the overset mesh can require an extensive amount of memory and the observed peak usage has been $90 \mathrm{~GB}$ on a single node, if seven compute nodes are used in total.

\section{Results}

Within this section, selected results of the numerical flow simulations dealing with the isolated RACER rotor head are presented. The performance of the newly developed blade-sleeve fairing (BSF) is determined by examining the aerodynamic forces acting on the rotor head. For this purpose, the obtained lift and drag are compared to a reference BSF, which was developed at Airbus Helicopters during the preliminary design phase of the new compound helicopter. This reference fairing offers a design, which is robust to reasonable changes of trim, as well as to flow inflection changes usually experienced during the upper-deck design convergence. Moreover, it will be used for the first flight of the RACER demonstrator. However, the new BSF was specifically optimized for the RACER cruise flight and therefore, a performance benefit is expected compared to the baseline fairing. Figure 12 shows the two rotor head fairings addressed within this publication. 


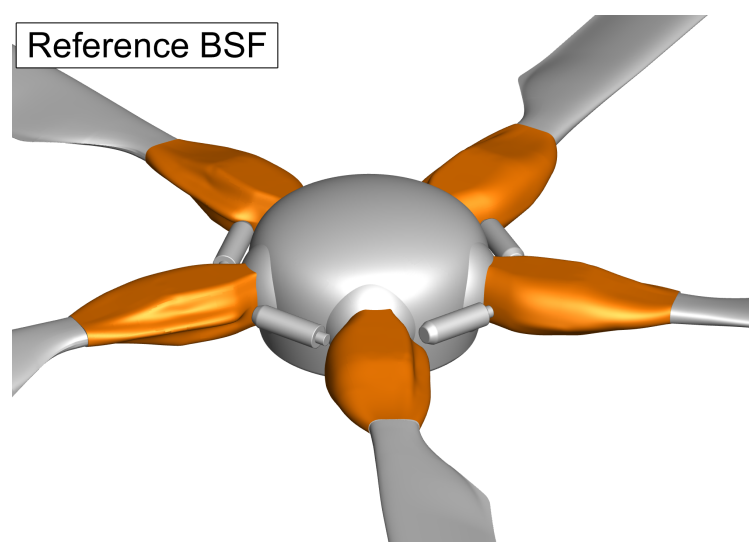

(a) Reference blade-sleeve fairing.

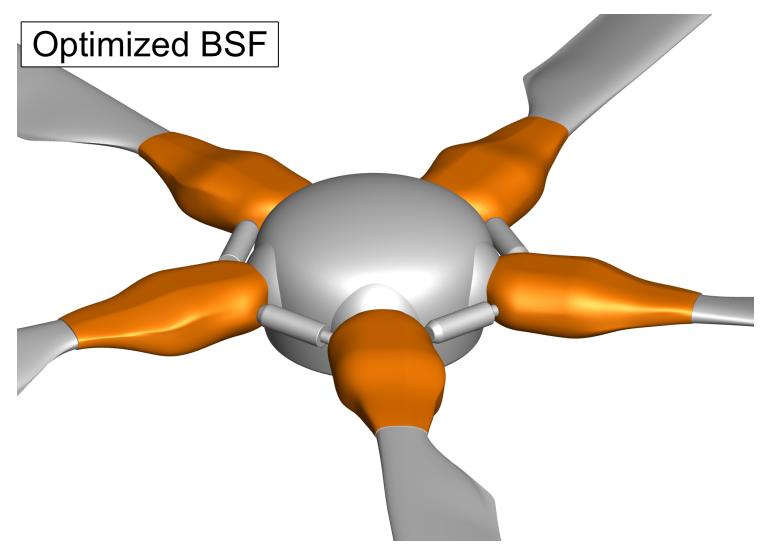

(b) Optimized blade-sleeve fairing.

Figure 12. Shape comparison between the reference blade-sleeve fairing and the optimized blade-sleeve fairing obtained within the FURADO project.

Besides the aerodynamic forces, the surface pressure distributions of the two BSFs are compared for the advancing $\left(\Psi=90^{\circ}\right)$ and the retreating $\left(\Psi=270^{\circ}\right)$ rotor blade position. These two azimuthal positions were considered during the design optimization of the new BSF, because the highest drag values are obtained on the sleeves under theses flow conditions. Finally, the flow field in the wake region of the RACER rotor head is presented giving an impression on the occurring flow phenomena.

\subsection{Aerodynamic Forces of the Isolated Rotor Head}

Regarding the aerodynamic forces, lift (L) and drag (D) are evaluated for the bladesleeve fairing (BSF), the full-fairing beanie (FFB) and the truncated rotor blade (RB). Overall, eight rotor revolutions have been simulated for each of the two configurations. At first, the aerodynamic forces acting on a single BSF are compared, which can be seen in Figure 13. All forces are normalized by the magnitude of the average force obtained for the reference BSF. The black curves correspond to the transient forces of the reference BSF and the green curves belong to the optimized BSF. Furthermore, the red dashed line shows the average value of the reference BSF, which is therefore -1 or 1 due to the normalization. The blue, dash-dotted lines yield the average forces of the optimized BSF. Figure 13a shows the lift for the final three rotor revolutions. By examining the average lift, it can be seen that, in general, the BSF causes a downforce. Regarding the optimized BSF, this downforce is reduced by $80 \%$ compared to the reference shape. A detailed view on a single rotor revolution $\left(0^{\circ} \leq \Psi \leq 360^{\circ}\right)$ is depicted in Figure 13b.

The largest discrepancies between the azimuthal lift distributions can be seen at $\Psi=90^{\circ}$, which corresponds to the advancing rotor blade position, and $\Psi=150^{\circ}$. Moreover, the interaction of the BSF with the unsteady wake flow field of the full-fairing beanie and the preceding rotor blade is reflected by the fluctuating lift forces between $\Psi=0^{\circ}$ and $\Psi=90^{\circ}$. Regarding the retreating rotor blade $\left(\Psi=270^{\circ}\right)$, reversed flow occurs and the normalized lift of the BSF is oscillating. The comparison of the normalized drag between the two different blade-sleeve fairings is shown in Figure 13c for three rotor revolutions and in Figure $13 \mathrm{~d}$ for a single rotor revolution. The predicted average drag of the optimized BSF could be reduced by $4.4 \%$. The largest drag values are observed at the advancing $\left(\Psi=90^{\circ}\right)$ and close to the retreating rotor blade position $\left(\Psi=270^{\circ}\right)$. Due to the increased dynamic pressure at the advancing rotor blade, the maximum drag is reached at this azimuthal position and it is about three times larger than the average drag. Comparing the drag force history of the optimized BSF with the reference fairing, only minor differences can be determined. At the upwind facing rotor blade $\left(\Psi=180^{\circ}\right)$, negative drag values can be observed, which means that the BSF is producing a small amount of thrust at this azimuthal position. The lowest normalized drag value is reached by the optimized BSF at $\Psi=168^{\circ}$ with $D / D_{\text {Ref. }}=-0.63$. 
The present investigations revealed that the shape of the BSF has a noticeable impact on the aerodynamic performance of the full-fairing beanie (FFB), which can be seen in Figure 14. Here, the lift and drag of the FFB depending on the applied BSF are compared to each other for a single rotor revolution. The reference forces are determined by the FFB in combination with the reference BSF. The normalized lift of the FFB during one rotor revolution is illustrated in Figure 14a. The force history shows five distinct peaks, which are related to the fact that the RACER demonstrator has five rotor blades. The maximum lift of the FFB is obtained, when the rotor blade is about 10-20 degrees after the upstream pointing position (e.g., $\Psi=190-200^{\circ}$ ). Moreover, minimum lift is generated, if there is no rotor blade in front of the FFB leading to clean inflow. Compared to the reference rotor head, the average lift of the FFB in combination with the optimized BSF is reduced by $23.8 \%$. The comparison of the normalized drag for a single rotor revolution is shown in Figure 14b. It can be seen that the azimuthal locations of the maximum and minimum drag values correlate with the ones derived for the normalized lift of the FFB. The amplitude of the drag fluctuation during one rotor revolution is significantly reduced by the application of the optimized BSF. However, the proportional drag of the FFB is increased by $3 \%$ compared to the reference BSF.

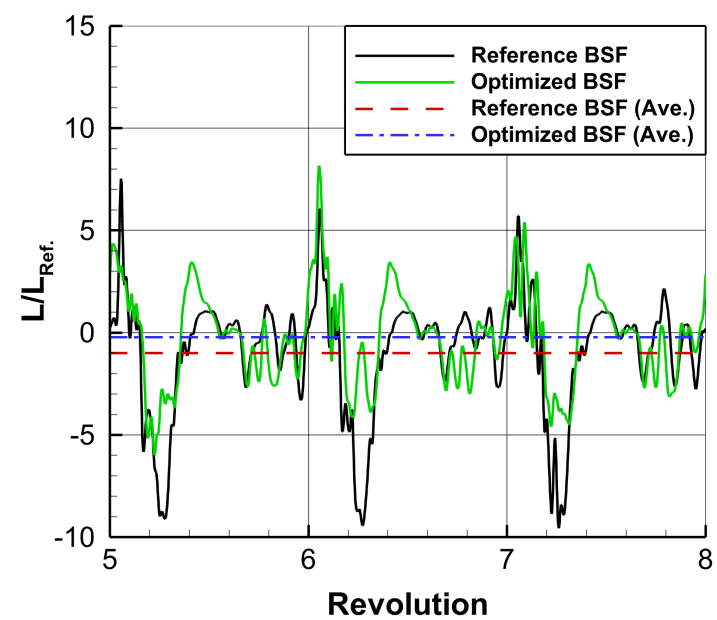

(a) Normalized lift $L / L_{\text {Ref. }}$. shown for three rotor revolutions.

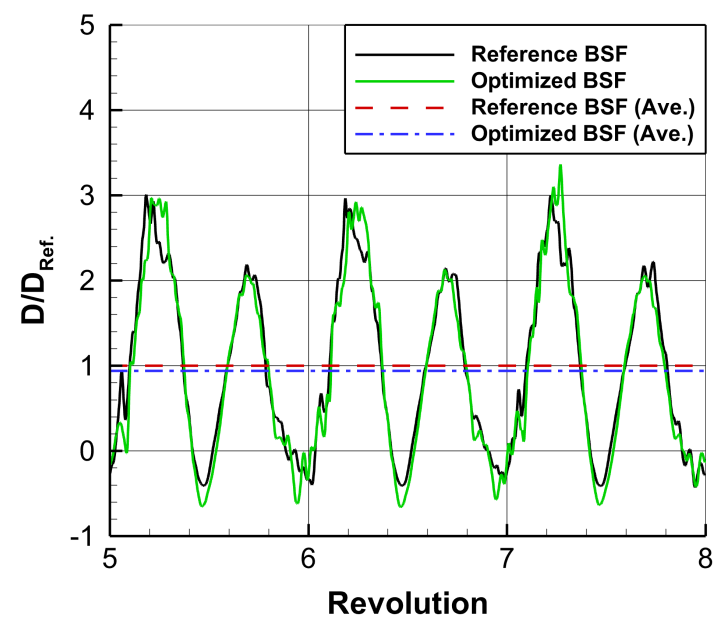

(c) Normalized drag $D / D_{\text {Ref. }}$ shown for three rotor revolutions.

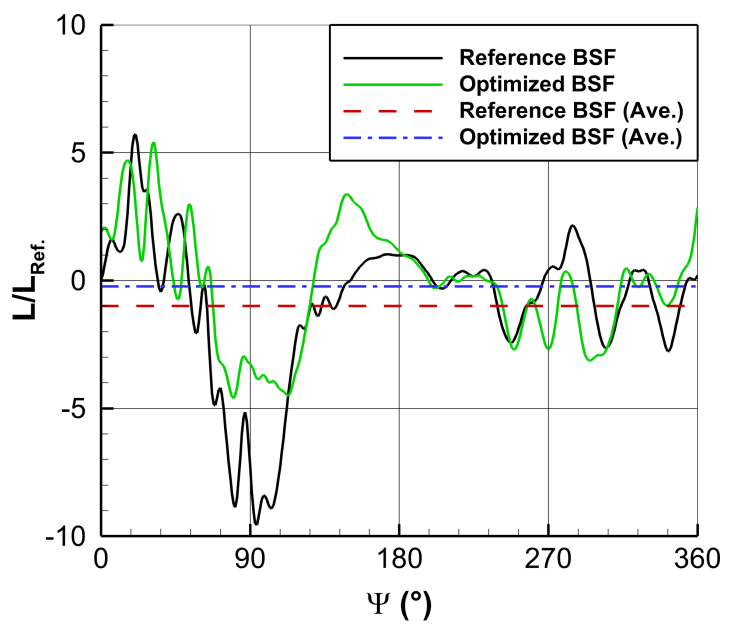

(b) Detailed view on the normalized lift $L / L_{\text {Ref }}$. depending on the azimuthal position $\left(0^{\circ} \leq \Psi \leq 360^{\circ}\right)$.

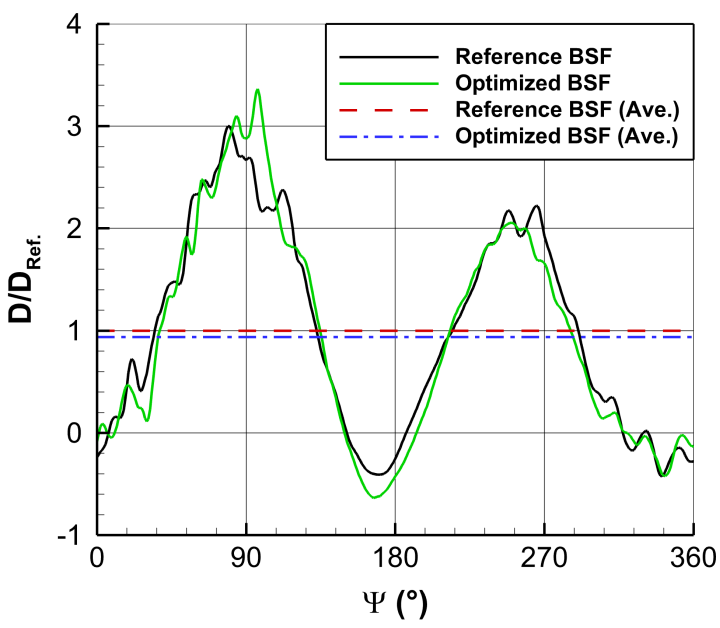

(d) Detailed view on the normalized drag $D / D_{\text {Ref. }}$ depending on the azimuthal position $\left(0^{\circ} \leq \Psi \leq 360^{\circ}\right)$.

Figure 13. Comparison of the aerodynamic forces between the optimized and the reference BSF. The forces are shown for a single BSF and they are normalized by the average values of the reference BSF. 


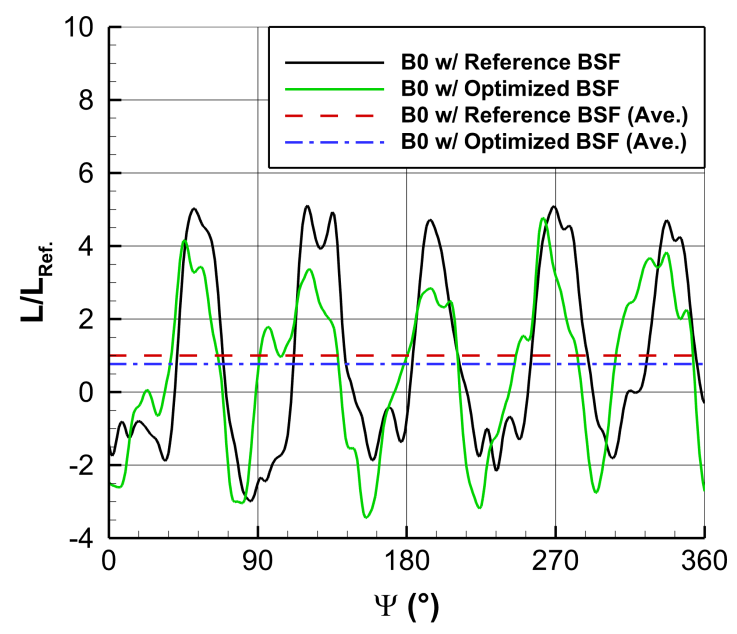

(a) Normalized lift $L / L_{\text {Ref. }}$.

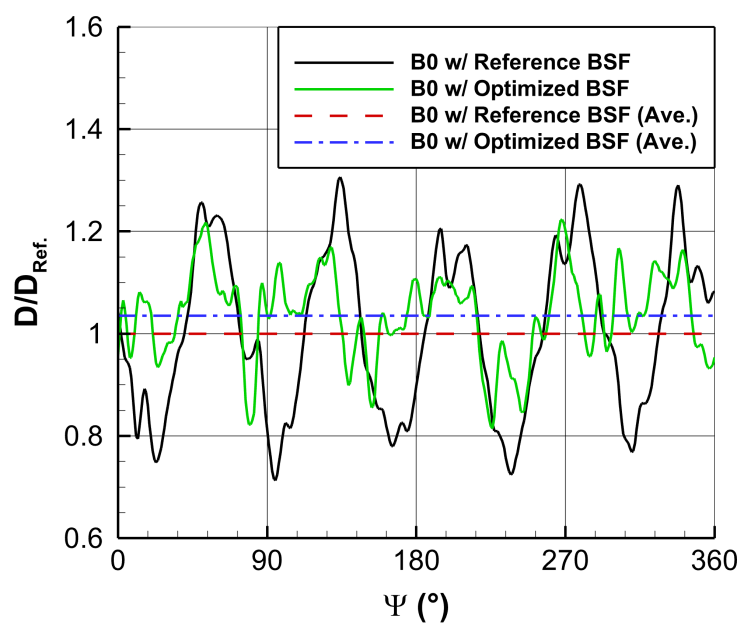

(b) Normalized drag $D / D_{\text {Ref. }}$.

Figure 14. Comparison of the normalized aerodynamic forces for the FFB in combination with the optimized and the reference BSF for a single rotor revolution.

Regarding the truncated rotor blades of the isolated rotor head, only little differences between the aerodynamic forces can be detected. Figure 15a shows the normalized lift of a single rotor blade stub for the final three rotor revolutions of the flow simulation. The force history between the two investigated rotor heads looks almost identical and the difference in the average lift is $5.8 \%$. A detailed view on the azimuthal lift distribution of one rotor revolution is given in Figure 15b. The minimum lift of the rotor blade stub is derived at the retreating blade position, which corresponds to $\Psi=270^{\circ}$. At this azimuthal position, reversed flow occurs and the rotor blade reaches its maximum pitch angle in cruise flight. In contrast, the maximum lift is obtained on the advancing blade side, which is related to the high dynamic pressure in this region. Even though the minimum blade pitch angle is reached at $\Psi \approx 90^{\circ}$ in cruise flight, the rotor blade stub still generates much more lift than on the retreating blade side. Figure 15c,d illustrate the comparison of the normalized drag. Regarding the rotor head with the optimized BSF, the drag contribution of the rotor blade stub is reduced by $2.4 \%$. Concerning Figure $15 \mathrm{~d}$, it can be seen that the normalized drag is matching very well within the range $135^{\circ}<\Psi<320^{\circ}$. The main differences are present within the advancing blade region, where interference with the preceding rotor blade is expected. There are two peak values at $\Psi=54^{\circ}$ and $\Psi=110^{\circ}$, which can be slightly reduced and smoothed by using the optimized BSF.

In order to be able to determine the performance benefit that can be achieved by the application of the optimized BSF, the total aerodynamic forces of the isolated rotor head are evaluated, which can be seen in Figure 16. For the sake of clarity, the transient forces are only shown for the rotor head with the optimized BSF. The normalized lift is illustrated in Figure 16a. The transient force of the rotor head with the optimized BSF is given by the green curve and the average lift is shown by the blue, dash-dotted line. Moreover, the minimum and maximum lift are marked by the black, dashed lines. Within the final three rotor revolutions a maximum lift of $L / L_{\text {Ref. }}=2.04$ and a minimum lift of $L / L_{\text {Ref. }}=0.54$ is reached. Regarding the reference rotor head, the red, dashed line shows the average lift. It can be seen that the rotor head with the optimized BSF achieves a lift increase of $20 \%$. Furthermore, Figure $16 \mathrm{~b}$ depicts the normalized drag of the isolated rotor head for the final three rotor revolutions. The same line style as for the normalized lift is used. The oscillation of the normalized drag is much smaller than for the normalized lift and it is varying within a range of $0.88 \leq D / D_{\text {Ref. }} \leq 1.03$. Compared to the reference rotor head, a drag reduction of $4.7 \%$ is achieved by the optimized BSF. 


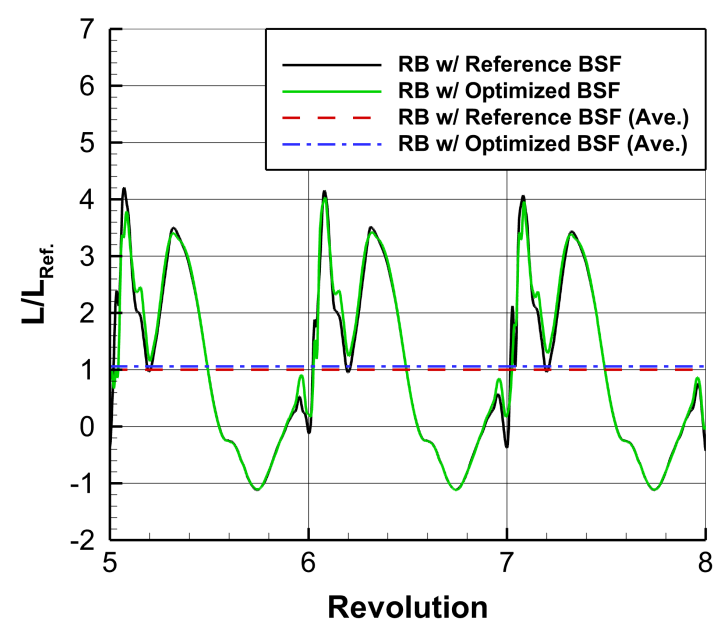

(a) Normalized lift $L / L_{\text {Ref. }}$. shown for three rotor revolutions.



(c) Normalized drag $D / D_{\text {Ref. }}$ shown for three rotor revolutions.



(b) Detailed view on the normalized lift $L / L_{\text {Ref. }}$ depending on the azimuthal position $\left(0^{\circ} \leq \Psi \leq 360^{\circ}\right)$.



(d) Detailed view on the normalized drag $D / D_{\text {Ref. }}$ depending on the azimuthal position $\left(0^{\circ} \leq \Psi \leq 360^{\circ}\right)$.

Figure 15. Comparison of the aerodynamic forces for a single rotor blade (RB) in combination with the optimized and the reference BSF. The forces are normalized by the average values of the RB combined with the reference BSF.

The evaluation of the transient aerodynamic forces revealed strong oscillations, especially concerning lift, and therefore, a fast Fourier transformation (FFT) is conducted to determine the dominant frequencies acting on the rotor head. The FFT of the normalized lift is shown in Figure 17 comparing the two investigated configurations. The frequency domain of the reference rotor head lift is illustrated in Figure 17a,b shows the spectrum of the optimized rotor head lift. The amplitudes of the oscillations are given relative to the reference forces and the frequency is shown in multiples of the blade-passing frequency (BPF). Regarding both investigated rotor heads, the first and the second harmonics of the BPF show relevant amplitudes in the same order of magnitude. The FFT of the rotor head drag revealed that only the first harmonic of the BPF is dominant for the reference rotor head, which is illustrated in Figure 18a. Concerning the rotor head with the optimized BSF, the oscillation at this frequency almost vanishes, which can be seen in Figure 18b. Table 3 summarizes the most relevant results of the FFT analysis showing the average values at $f / B P F=0$ and the first five BPF harmonics. 


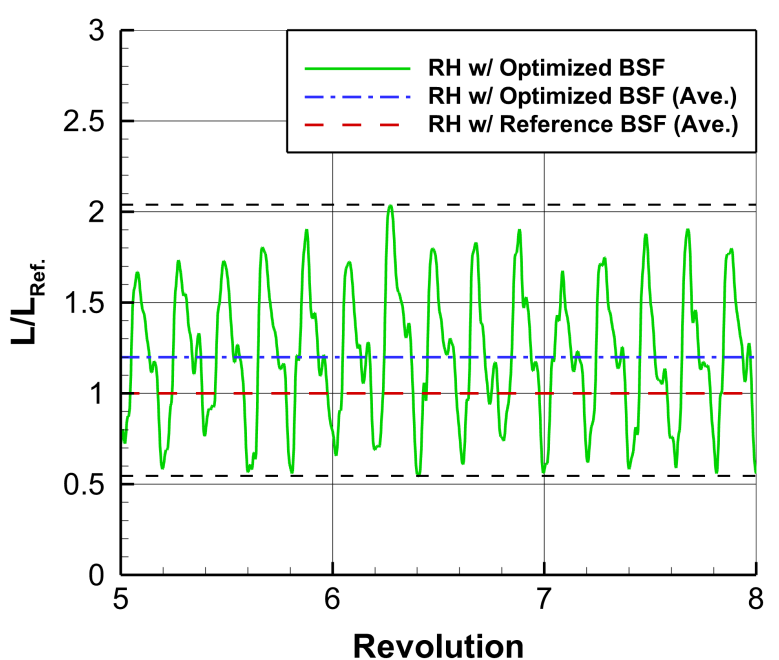

(a) Normalized lift $L / L_{\text {Ref. }}$ shown for three rotor revolutions.

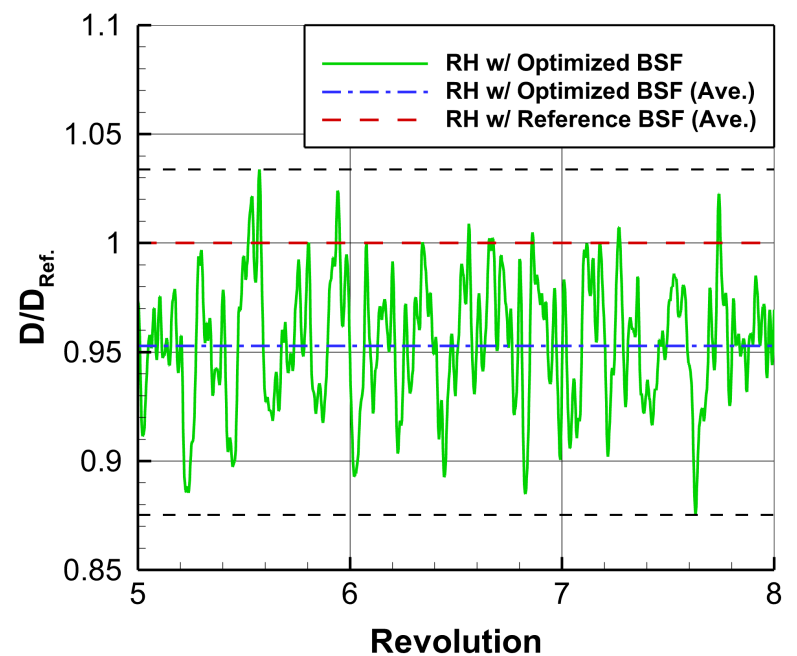

(b) Normalized drag $D / D_{\text {Ref. }}$ shown for three rotor revolutions.

Figure 16. Total lift and drag of the isolated rotor head for the final three rotor revolutions. The forces are normalized by the average values of the reference rotor head.

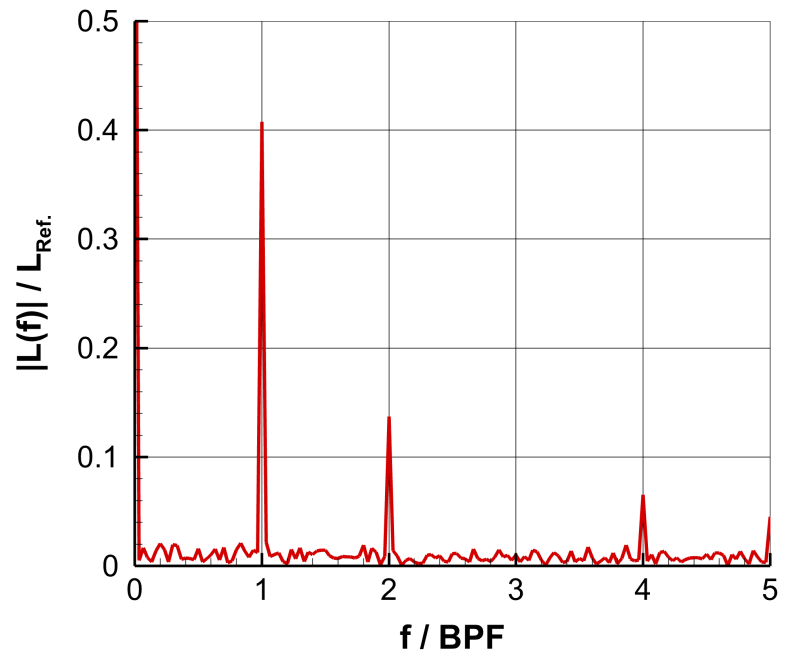

(a) Rotor head with the reference BSF.

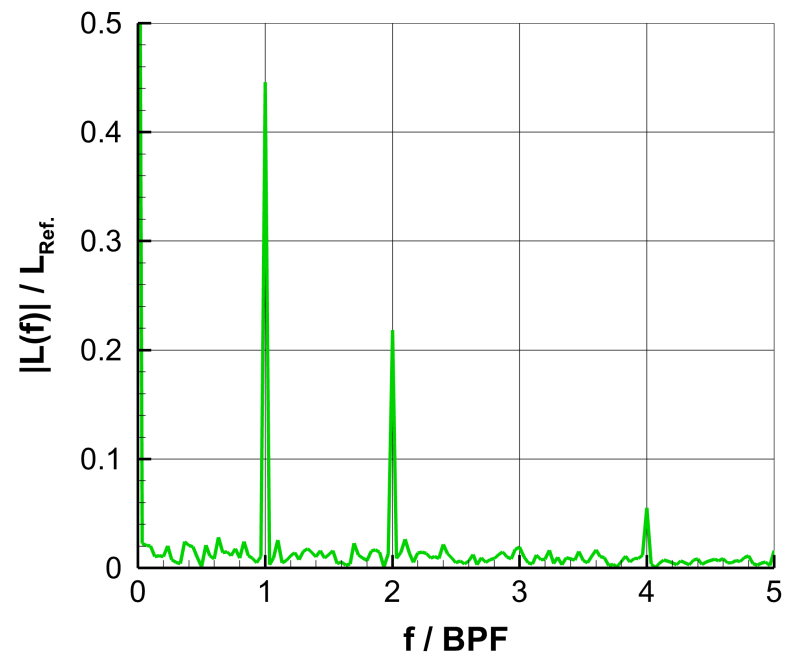

(b) Rotor head with the optimized BSF.

Figure 17. Spectrum of the rotor head lift showing dominant frequencies acting on the rotor head.

Table 3. Summary of the FFT analysis regarding rotor head lift and drag.

\begin{tabular}{ccccc}
\hline & \multicolumn{2}{c}{ RH w/ Reference BSF } & \multicolumn{2}{c}{ RH w/ Optimized BSF } \\
\hline$f / B P F$ & $|L(f)| / L_{\text {Ref. }}$ & $|\boldsymbol{D}(f)| / \boldsymbol{D}_{\text {Ref. }}$ & $|L(f)| / L_{\text {Ref. }}$ & $|\boldsymbol{D}(f)| / \boldsymbol{D}_{\text {Ref. }}$ \\
\hline 0 & 1.000 & 1.000 & 1.201 & 0.953 \\
1 & 0.408 & 0.112 & 0.446 & 0.028 \\
2 & 0.137 & 0.014 & 0.218 & 0.011 \\
\hline 3 & 0.010 & 0.011 & 0.019 & 0.006 \\
4 & 0.065 & 0.003 & 0.055 & 0.004 \\
5 & 0.045 & 0.007 & 0.016 & 0.004 \\
\hline
\end{tabular}




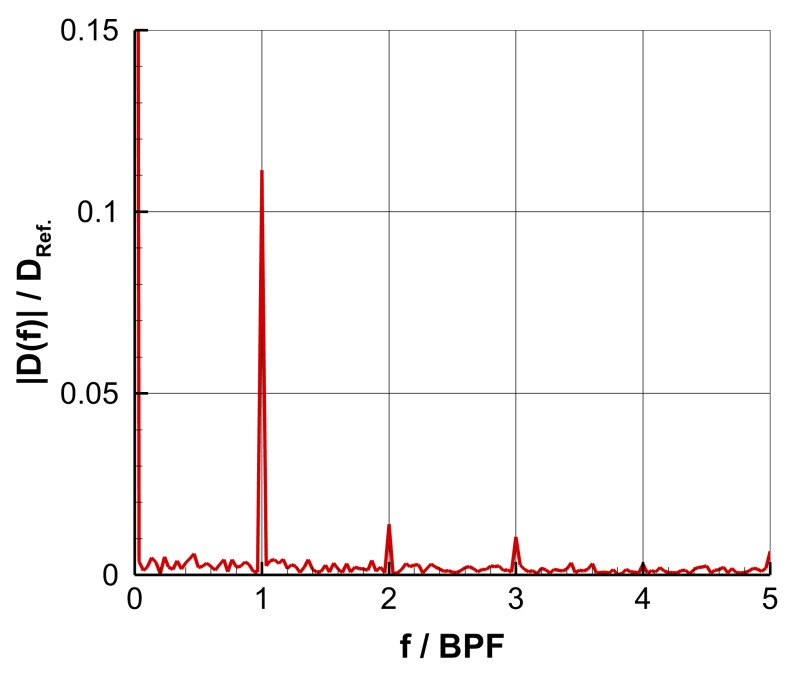

(a) Rotor head with the reference BSF.

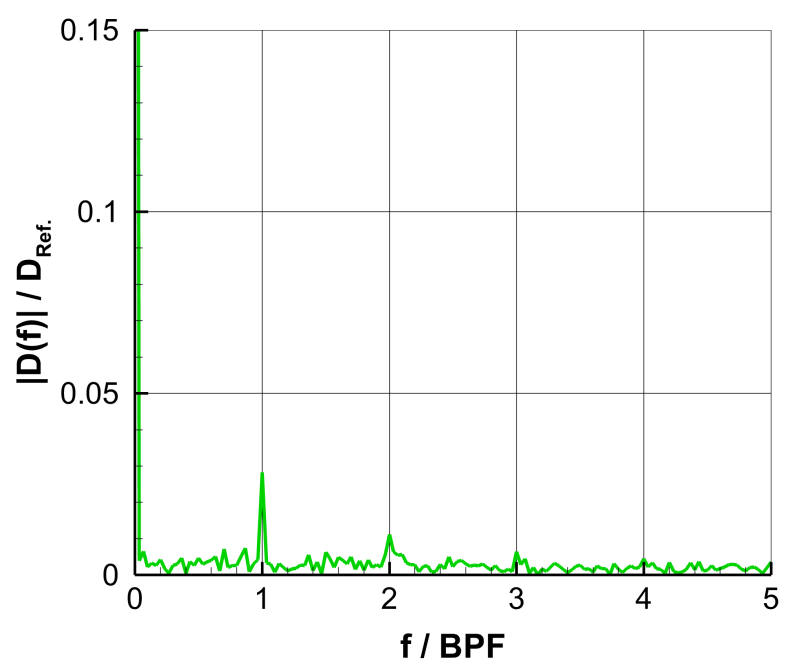

(b) Rotor head with the optimized BSF.

Figure 18. Spectrum of the rotor head drag showing dominant frequencies acting on the rotor head.

\subsection{Surface Pressure Distribution}

The design optimization of the BSF shape was performed for a single, steady rotor blade considering the advancing and the retreating rotor blade position. Therefore, these two azimuthal positions are considered for the evaluation of the surface pressure distribution within the present work. Moreover, the chord-wise pressure distribution is examined in four radial BSF sections (S1-S4). These sections were aerodynamically optimized within the FURADO project and they represent the main supporting structure defining the shape of the BSF [22]. The comparison of the surface pressure distribution between the optimized and the reference BSF is illustrated in Figure 19 for the advancing rotor blade $\left(\Psi=90^{\circ}\right)$ and in Figure 20 for the retreating rotor blade $\left(\Psi=270^{\circ}\right)$. Both figures include a contour plot of the surface pressure coefficient $C_{p}$ and four chord-wise pressure distributions $C_{p}(x / c)$ shown at the Sections S1-S4. The pressure coefficient $C_{p}$ is normalized by the total pressure coefficient $C_{p t, S 1}$ obtained at the stagnation point of the first radial section $S 1$, for the advancing and the retreating rotor blade, respectively. Furthermore, the pressure distribution corresponding to the lower side of the BSF is marked by " $\mathrm{L}$ " and for the upper side it is marked by "U".

The pressure distributions in the radial sections S1-S3 of the advancing reference BSF, which can be seen in Figure 19a, reveal that flow separation is triggered at $x / c=0.75-0.8$ due to a distinct edge in the contour of the reference BSF. The region of separated flow is defined by a constant pressure plateau downstream of $x / c=0.75$. Furthermore, in the front part of the fairing, the pressure on the upper side is higher than on the lower side, which causes a downforce. This negative pressure balance can be observed up to $x / c=0.6-0.65$. Thereafter, a pronounced suction peak is present on the upper side of the fairing, which is located at about $73 \%$ of the chord length.

This suction peak leads to a positive pressure balance between the upper the lower side generating a small amount of lift in the back part of the fairing. This effect becomes stronger for the optimized BSF, especially regarding Sections S2 and S3, which can be seen in Figure 19b. Additionally, the pressure distribution in section S1 indicates a flow separation at $x / c=0.8$. Regarding Section S2 and S3, it seems that the flow separation could be delayed to $x / c=0.95$ or even further. However, the pressure distribution can only indicate a region of flow separation and for detailed analysis the wall shear stress must be considered. Regarding both BSFs, the largest pressure differences between the upper and lower side are obtained in the front part of section S4. Regarding Sections S1 and S2 of the optimized BSF, the pressure difference in the front part could be reduced compared to the reference BSF, which causes less downforce in this region of the fairing. 


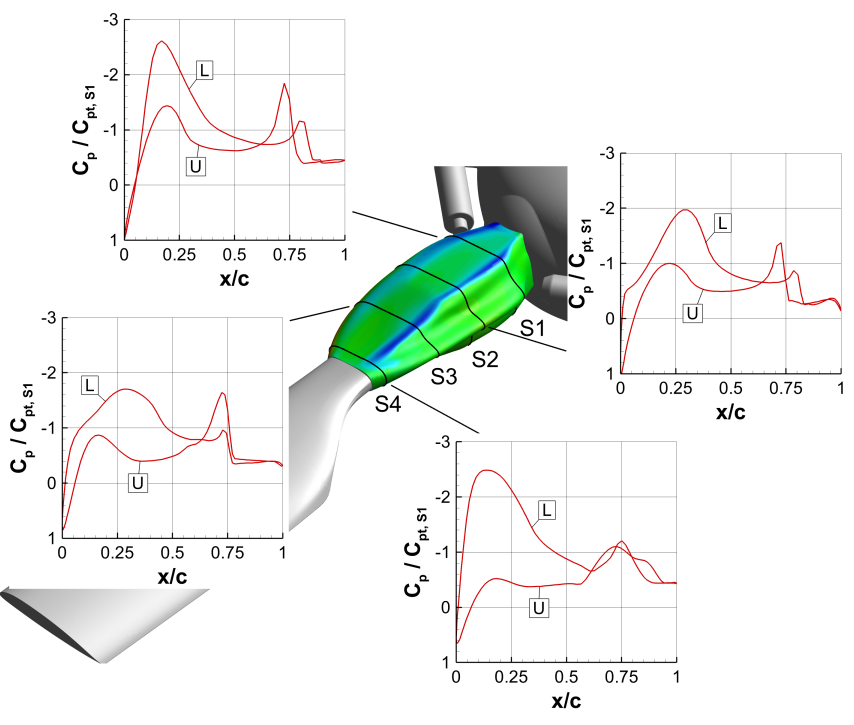

(a) Reference BSF.

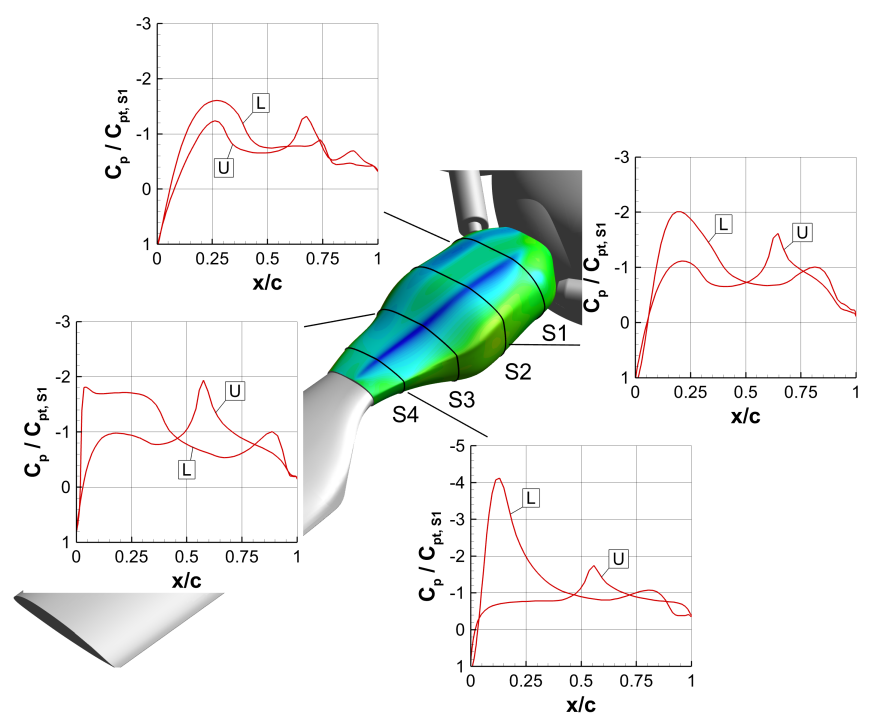

(b) Optimized BSF.

Figure 19. Comparison of the normalized, chord-wise surface pressure distribution $C_{p} / C_{p t, S 1}(x / c)$ at four radial sections S1-S4 considering the advancing rotor blade position $\left(\Psi=90^{\circ}\right)$.

The comparison of the surface pressure distribution for the retreating rotor blade $\left(\Psi=270^{\circ}\right)$ is depicted in Figure 20. At this azimuthal position, reversed flow occurs, because the circumferential speed of the BSF is much smaller than the cruise speed of the helicopter. Therefore, a region of stagnated flow can be seen on the backside of the fairing and the sectional pressure distributions seem mirrored compared to the advancing blade case. Moreover, the rotor blade reaches its maximum pitch angle at this azimuth, which, in combination with the reversed flow in the inboard region, leads to a negative angle of attack for the incoming flow. As already observed for the advancing rotor blade, the pressure on the upper side of the BSF is usually higher than on the lower side, which in total generates a downforce. However, this is not the case for Section S1 of the reference $\mathrm{BSF}$, which provides a positive pressure balance generating lift in this region. Comparing the sectional pressure distributions of the two different fairings for the Sections S1-S3, it can be seen that the suction peaks within the region $0.65 \leq x / c \leq 0.75$ are much more pronounced for the reference BSF than for the optimized BSF. Regarding the pressure recovery at $x / c=0$, similar pressure levels can be observed for the Sections S3 and S4 of both fairings. In Section S1, a better pressure recovery is predicted for the optimized BSF showing a pressure difference of $\Delta C_{p} / C_{p t, S 1}=+0.4$. Moreover, in Section S2 of the optimized BSF, a pressure jump can be observed at $x / c \approx 0$, which is related to a vortical flow structure shedding of the blade-sleeve fairing and interacting with the surface in this region at the current time step.

\subsection{Flow Field Visualization}

In order to give an impression of the prevailing flow field in the vicinity of the isolated rotor head, selected flow field quantities are presented within this section. The results of the rotor head with the optimized BSF are directly compared to the ones obtained for the reference fairing. Figure 21 shows the normalized axial flow velocity $u / V_{\infty}$ in seven equally spaced slices downstream of the rotor head. All values above $0.99 \cdot u / V_{\infty}$ are cut off to demonstrate the velocity deficit introduced by the presence of the isolated rotor head. Regions of stagnated or even reversed flow can only be detected right after the beanie and close to the BSF of the advancing rotor blade. Furthermore, only minor discrepancies between the two investigated rotor heads can be seen and a similar recovery of the axial flow velocity can be observed. 


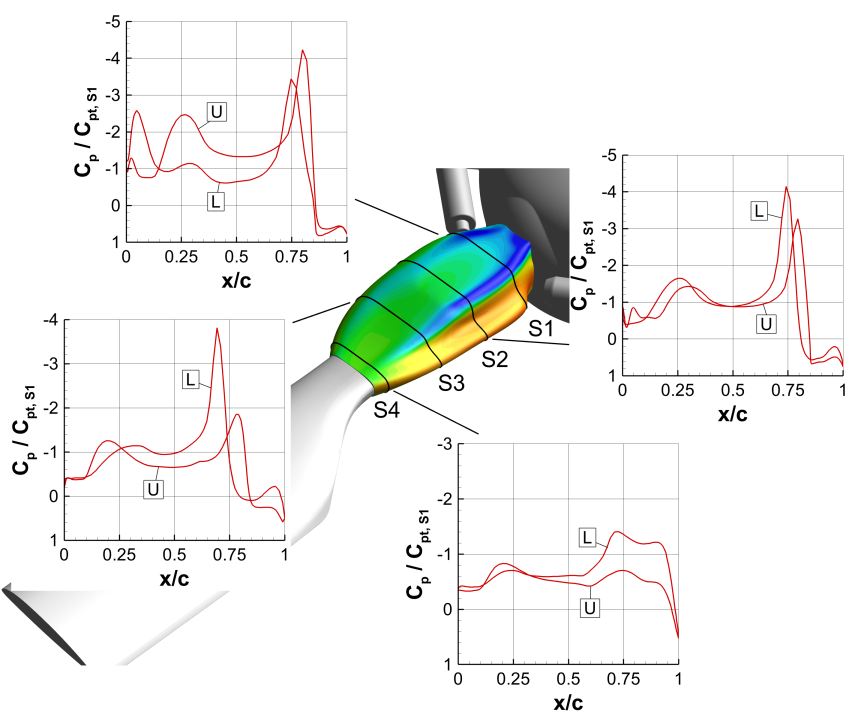

(a) Reference BSF.

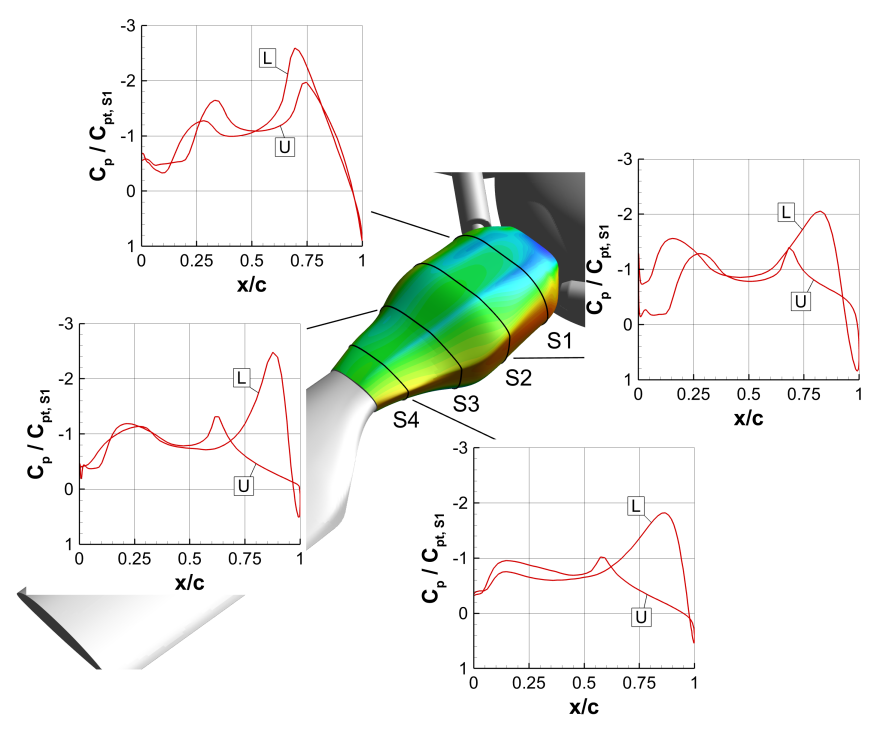

(b) Optimized BSF.

Figure 20. Comparison of the normalized, chord-wise surface pressure distribution $C_{p} / C_{p t, S 1}(x / c)$ at four radial sections S1-S4 considering the retreating rotor blade position $\left(\Psi=270^{\circ}\right)$.

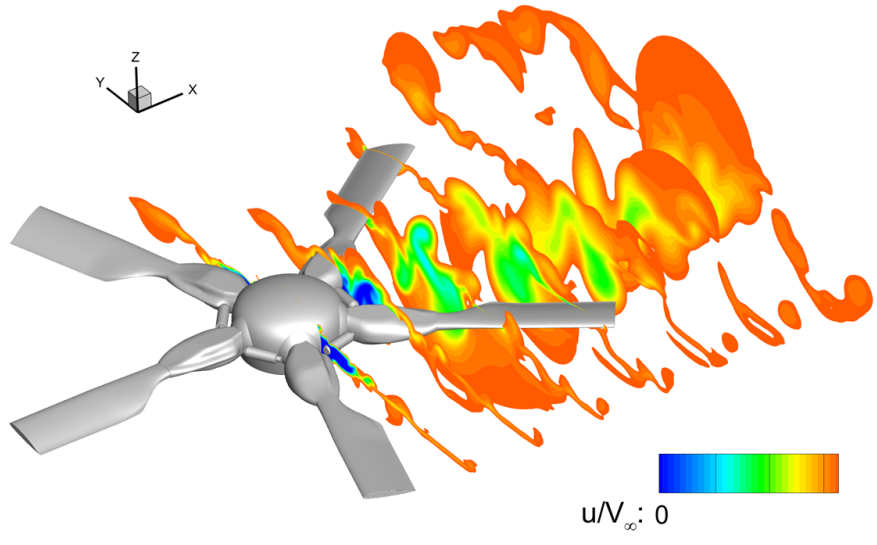

(a) Rotor head with the reference BSF.

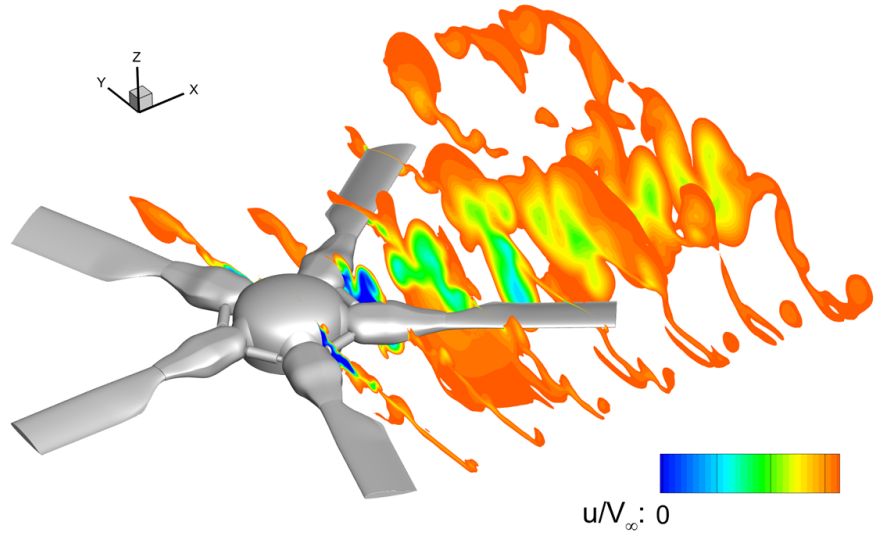

(b) Rotor head with the optimized BSF.

Figure 21. Normalized axial flow velocity $u / V_{\infty}$ shown in seven equally spaced slices in the wake region of the rotor head. All velocities above $0.99 u / V_{\infty}$ are cut off.

Figure 22 shows the normalized axial vorticity $\omega_{x} \cdot R / V_{\infty}$ in equally spaced slices positioned along the flow direction. In the wake region of the full-fairing beanie, a large number of vortical flow structures and discrete vortices can be detected. Furthermore, the blade tip vortices of the upwind facing and the advancing rotor blade, which are characterized by a negative axial vorticity, can cleary be observed. It can be seen that the blade tip vortex of the upwind facing rotor blade is interacting with the advancing rotor blade, which influences the surface pressure distribution in this region. Moreover, the two blade tip vortices are merging further downstream. Regarding the vortical flow structures in the wake region of the full-fairing beanie, strong interaction effects can be seen and the vorticity magnitude is rapidly decreasing in downstream direction. 


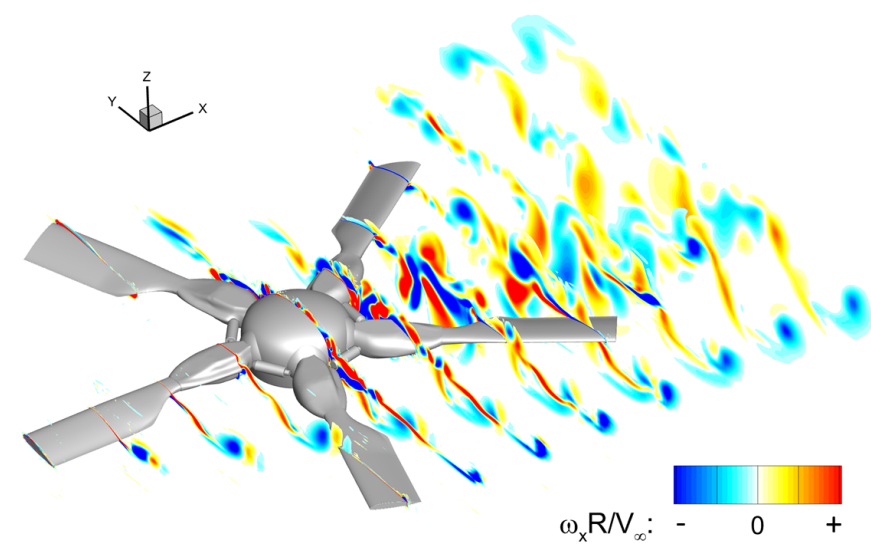

(a) Rotor head with the reference BSF.

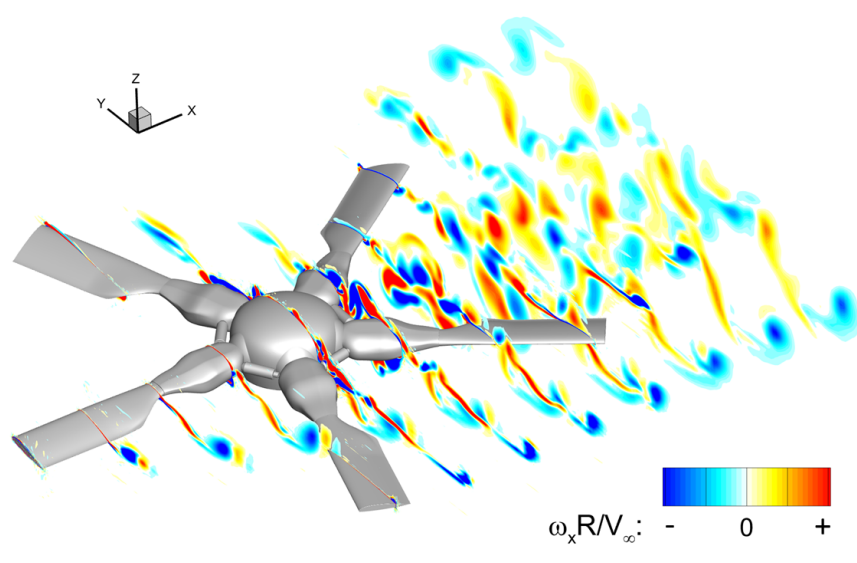

(b) Rotor head with the optimized BSF.

Figure 22. Normalized axial vorticity $\omega_{x} \cdot R / V_{\infty}$ shown in ten equally spaced slices along the flow direction.

In order to complement the visualization of the axial vorticity, shown in Figure 22, an iso-surface of the Q-criterion with $Q=2000 \mathrm{~s}^{-2}$ is depicted in Figure 23 comparing the reference and the optimized rotor head. Moreover, the iso-surface is colored by the normalized axial flow velocity $u / V_{\infty}$. By means of this vortex identification criterion, the complexity of the highly turbulent wake flow field can be demonstrated. Moreover, regions of stagnated flow can be identified by the blue colored regions in Figure 23. The red surfaces show regions of increased axial flow velocity, which can be observed on top of the full-fairing beanie, the advancing rotor blade and the retreating blade-sleeve fairing. Furthermore, the previously mentioned blade-tip vortices of the upwind facing and the advancing rotor blade are well captured by the Q-criterion.

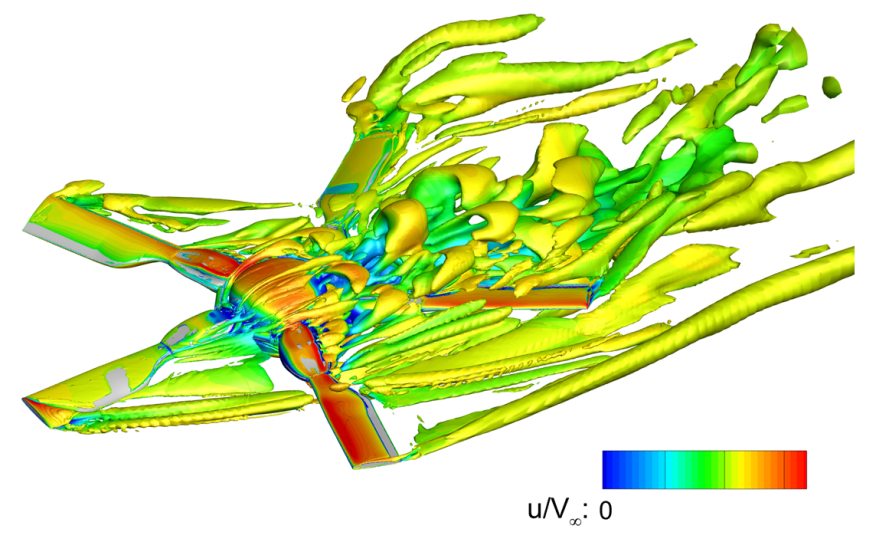

(a) Rotor head with the reference BSF.



(b) Rotor head with the optimized BSF.

Figure 23. Iso-surface of the $Q$-criterion $\left(Q=2000 s^{-2}\right)$, which is colored by the normalized axial flow velocity $u / V_{\infty}$.

\section{Conclusions}

In the course of the Clean Sky 2 project FURADO, a blade-sleeve fairing was aerodynamically optimized for a sea-level cruise flight of the RACER compound helicopter. Within the present publication, the performance of the newly developed fairing is evaluated on the isolated, five-bladed RACER rotor head. For this purpose, unsteady numerical flow simulations are performed considering a full-scale model of the rotating rotor head with cyclic pitch movement. The main focus of the present work is on the evaluation of the aerodynamic forces acting on the rotor hub. The cyclic pitch movement of the rotor blades is realized by an overset mesh approach in combination with mesh motion. In order to be able to assess the performance of the optimized blade-sleeve fairing, it is compared to a reference fairing, which was developed at Airbus Helicopters during the preliminary design phase of the RACER demonstrator. The 
transient and average forces are evaluated for the full-fairing beanie, the blade-sleeve fairing and the truncated rotor blade. Regarding the aerodynamic forces acting on the blade-sleeve fairing itself, the downforce could be decreased by $79.5 \%$ and a drag reduction of $4.4 \%$ could be achieved in comparison to the reference blade-sleeve fairing. Considering the total rotor head forces including all components and therefore, all interference effects, a lift increase of $20 \%$ and a drag reduction of $4.7 \%$ could be obtained on the isolated rotor head with the optimized blade-sleeve fairing. Additionally, an FFT analysis was conducted for the transient lift and drag to determine dominant frequencies and amplitudes acting on the rotor head. Concerning lift, the first and the second harmonics of the blade-passing frequency caused noteworthy amplitudes for both, the optimized and the reference rotor head. Regarding drag, only the reference rotor head showed a relevant oscillation for the first harmonic of the blade-passing frequency. In order to determine the main reasons for the performance improvement achieved by the optimized blade-sleeve fairing, the surface pressure distribution is evaluated and compared to the reference blade-sleeve fairing. Furthermore, the occurring flow phenomena in the vicinity and the wake region of the rotor head are visualized by selected flow field quantities.

Author Contributions: Conceptualization, P.P., D.D. and C.B.; methodology, P.P. and A.K.; numerical setup, P.P. and A.K.; flow simulation, P.P.; investigation and analysis, P.P., draft preparation, P.P.; review and editing, P.P.; visualization, P.P.; supervision, C.B. and D.D.; project administration, C.B.; funding acquisition, C.B. All authors have read and agreed to the published version of the manuscript.

Funding: The research leading to these results is part of the FURADO project. This project received funding from the European Union within the Clean Sky 2 framework and under the grant agreement number 685636.

Acknowledgments: Special thanks are addressed to ANSYS for providing the flow simulation software. The authors gratefully acknowledge the Gauss Centre for Supercomputing e.V. (www. gauss-centre.eu (accessed on 18 January 2021)) for funding this project by providing computing time on the GCS Supercomputer SuperMUC-NG at Leibniz Supercomputing Centre (www.lrz.de (accessed on 18 January 2021)).

Conflicts of Interest: The authors declare no conflict of interest.

\section{Abbreviations}

The following abbreviations are used in this manuscript:

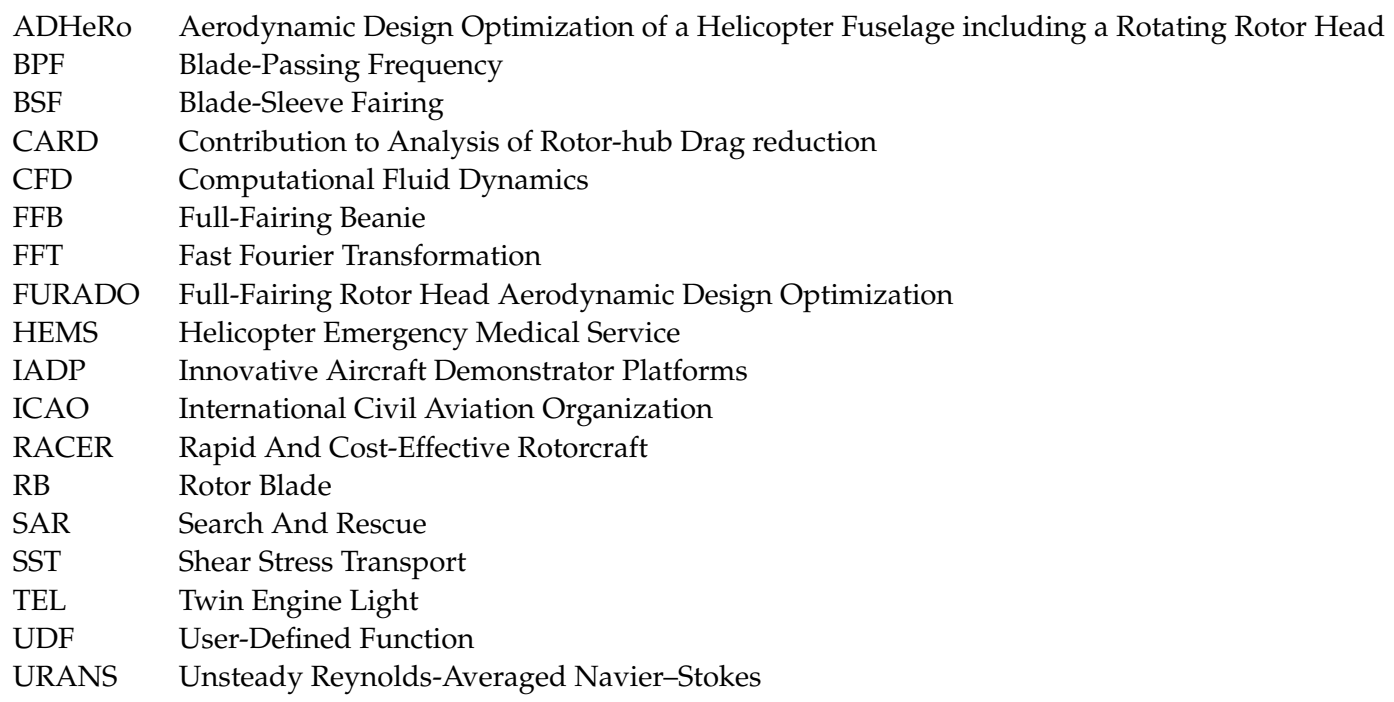

\section{References}

1. IATA's Annual Review. 2019. Available online: https://www.iata.org/en/publications/annual-review/ (accessed on 9 December 2020).

2. Flightpath 2050—Europe's Vision for Aviation. Available online: https://ec.europa.eu/transport/sites/transport/files/modes/ air/doc/flightpath2050.pdf (accessed on 9 December 2020). 
3. Clean Sky. Available online: https://www.cleansky.eu/ (accessed on 9 December 2020).

4. Johnson, W. Maximum Speed. In Helicopter Theory; Dover Publications Inc.: Mineola, NY, USA, 1994; pp. $297-298$.

5. Airbus Helicopters Reveals Racer High-Speed Demonstrator Configuration. Available online: https://www.airbus.com/ newsroom/press-releases/en/2017/06/Airbus-Helicopters-reveals-Racer-high-speed-demonstrator-configuration.html (accessed on 9 December 2020).

6. Stalewski, W.; Zoltak, J. Optimisation of the Helicopter Fuselage with Simulation of Main and Tail Rotor Influence. In Proceedings of the 28th International Congress of the Aeronautical Sciences, Brisbane, Australia, 23-28 September 2012.

7. Batrakov, A.S.; Kusyumov, A.N.; Mikhailov, S.A.; Barakos, G.N. Aerodynamic Optimization of Helicopter Rear Fuselage. Aerosp. Sci. Technol. 2018, 77, 704-712. [CrossRef]

8. Le Pape, A.; Lienard, C.; Verbeke, C.; Pruvost, M.; De Coninck, J.-L. Helicopter Fuselage Drag Reduction using Active Flow Control: A Comprehensive Experimental Investigation. J. Am. Helicopter Soc. 2015, 60, 1-12. [CrossRef]

9. De Gregorio, F. Helicopter Fuselage Model Drag Reduction by Active Flow Control Systems. In Proceedings of the 43rd European Rotorcraft Forum, Milan, Italy, 12-15 September 2017.

10. Reß, R.; Grawunder, M.; Breitsamter, C. Parasite Drag Reduction of a Twin Engine Lightweight Helicopter Configuration. In Proceedings of the 63rd German Aerospace Congress, Augsburg, Germany, 16-18 September 2014.

11. Grawunder, M.; Reß, R.; Breitsamter, C. Helicopter Aft-Body Drag Reduction by Passive Flow Control. In Proceedings of the 40th European Rotorcraft Forum, Southampton, UK, 2-5 September 2014.

12. Boniface, J.-C. A Computational Framework for Helicopter Fuselage Drag Reduction using Vortex Generators. J. Am. Helicopter Soc. 2016, 61, 1-13. [CrossRef]

13. Wagner, S. Problems of estimating the drag of a helicopter. In Proceedings of the 124th AGARD Conference, Izmir, Turkey, 10-13 April 1973.

14. Grawunder, M.; Reß, R.; Breitsamter, C.; Adams, N.A. Flow characteristics of a helicopter fuselage configuration including a rotating rotor head. In Proceedings of the 28th International Congress of the Aeronautical Sciences, Brisbane, Australia, 23-28 September 2012.

15. Desvigne, D.; Alfano, D. Rotor-head/Fuselage Interactional Effects on Helicopter Drag: Influences of the Complexification of the Rotor-head Geometry. In Proceedings of the 39th European Rotorcraft Forum, Moscow, Russia, 3-6 September 2013.

16. Khier, W. Numerical Analysis of Hub and Fuselage Interference to reduce Helicopter Drag. In Proceedings of the 38th European Rotorcraft Forum, Amsterdam, The Netherlands, 4-7 September 2012.

17. Desvigne, D.; Alfano, D. Helicopter Drag Mitigation during the CARD Project: Numerical Investigation of Rotor and Pylon Fairings. In Proceedings of the 42nd European Rotorcraft Forum, Lille, France, 5-8 September 2016.

18. Graham, D.R.; Sung, D.Y.; Young, L.A.; Louie, A.W.; Stroub, R.H. Helicopter Hub Fairing and Pylon Interference Drag; NASA Technical Memorandum 101052; Ames Research Center: Moffett Field, CA, USA, 1989.

19. Martin, D.M.; Mort, R.W.; Young, L.A.; Squires, P.K. Experimental Investigation of Advanced Hub and Pylon Fairing Configurations to Reduce Helicopter Drag; NASA Technical Memorandum 4540; Ames Research Center: Moffett Field, CA, USA, 1993.

20. Wentrup, M.; Khier, W.; Zhang, Q. Drag Reduction of a Transport Helicopter by Application of an adjoint-based Fuselage Optimization Chain and Modification of the Rotor Head. In Proceedings of the Greener Aviation Conference: Clean Sky breakthroughs and worldwide status, Brussels, Belgium, 12-14 March 2014.

21. Full Fairing Rotor Head Aerodynamic Design Optimization (FURADO). Available online: www.furado.tum.de (accessed on 19 December 2020).

22. Pölzlbauer, P.; Desvigne, D.; Breitsamter, C. Aerodynamic Design Optimization of a Helicopter Rotor Blade-Sleeve Fairing. CEAS Aeronaut. J. 2019, 10, 665-685. [CrossRef]

23. Pölzlbauer, P.; Desvigne, D.; Breitsamter, C. Performance Improvement of a Compound Helicopter Rotor Head by Aerodynamic Design Optimization of a Blade-Sleeve Fairing. Aeronaut. J. 2019, 123, 1456-1475. [CrossRef]

24. Airbus Helicopters H175 Main Rotor Head. Available online: https://gallery.vtol.org/image/EtSD (accessed on 1 November 2020).

25. Benek, J.A.; Steger, J.L.; Dougherty, F.C. A Flexible Grid Embedding Technique with Application to the Euler Equations. In Proceedings of the 6th AIAA Computational Fluid Dynamics Conference, Danvers, MA, USA, $13-15$ July 1983.

26. Benek, J.A.; Steger, J.L.; Dougherty, F.C.; Buning, P.G. Chimera: A Grid-Embedding Technique; Final Technical Report AEDC-TR-85-64; Arnold Engineering Development Center, Air Force Systems Command Arnold Air Force Station: Tullahoma, TN, USA, 1986.

27. Lombard, C.K.; Venkatapathy, E. Implicit boundary treatment for joined and disjoint patched mesh systems. In Proceedings of the 7th AIAA Computational Physics Conference, Cincinnati, OH, USA, 15-17 July 1985.

28. ANSYS, Inc. ANSYS Fluent User's Guide; Release 2019.R2; ANSYS, Inc.: Canonsburg, PA, USA, 2019.

29. Menter, F.R. Two-Equation Eddy-Viscosity Turbulence Models for Engineering Applications. AIAA J. 1994, 32, 1598-1605. [CrossRef] 